\title{
Spectral and Scattering Theory for Schrödinger Operators with Cartesian Anisotropy
}

\author{
By
}

Serge RICHARD*

\begin{abstract}
We study the spectral and scattering theory of some $n$-dimensional anisotropic Schrödinger operators. The characteristic of the potentials is that they admit limits at infinity separately for each variable. We give a detailed analysis of the spectrum: the essential spectrum, the thresholds, a Mourre estimate, a limiting absorption principle and the absence of singularly continuous spectrum. Then the asymptotic completeness is proved and a precise description of the asymptotic states is obtained in terms of a suitable family of asymptotic operators.
\end{abstract}

\section{$\S 1$. Introduction}

In this paper we shall be interested in the spectral and scattering theory of some anisotropic Schrödinger operators $H=-\boldsymbol{\Delta}+V$ in the Hilbert space $L^{2}\left(\mathbb{R}^{n}\right)$. A general theory for highly anisotropic potentials is still lacking, but various partial approaches are already well developed. The most famous one, and best achieved, is with no doubt the N-body problem (see [16], [6], [18] and [4]). Let us also mention [8] and [12] for the spectral analysis of general anisotropic systems, [3] for the scattering theory for systems with different spatial asymptotics on the left and right, and [10] and references therein for a thorough analysis of Schrödinger operators with potentials independent of $|x|$. Here another type of anisotropy is considered. It is called cartesian since the potentials $V$ admit limits at infinity separately for each variable. For the

Communicated by T. Kawai. Received May 14, 2003. Revised October 6, 2003.

2000 Mathematics Subject Classification(s): 35P25, 47C15, 81Q10.

Key words: Schrödinger operator, spectral and scattering theory, anisotropic potential, compactification, crossed product, asymptotic velocity, minimal velocity.

*Department of Theoretical Physics, University of Geneva, 1211 Geneva 4, Switzerland. e-mail: serge.richard@physics.unige.ch 
corresponding operators, the spectral and scattering theory can be completely achieved. Moreover, since our approach to the propagation properties of states is close to intuition, we expect that it could stimulate the development of a general theory.

Let us illustrate our framework with a simple example. We consider the operator $H=-\boldsymbol{\Delta}+V$ in $L^{2}\left(\mathbb{R}^{2}\right)$, with $V\left(x_{1}, x_{2}\right)=V_{1}\left(x_{1}\right) V_{2}\left(x_{2}\right)$, and for $j \in\{1,2\}, V_{j}$ is a continuous real function defined on $\mathbb{R}$ which has limits $c_{j}^{ \pm}$ at $\pm \infty$ and converges to these limits in a short-range way. We call asymptotic Hamiltonians the operators $H_{j \pm}=-\boldsymbol{\Delta}+c_{k}^{ \pm} V_{j}$, with $j, k \in\{1,2\}$ but $j \neq$ $k$, and internal Hamiltonians the operators $H^{j \pm}=-\boldsymbol{\Delta}_{j}+c_{k}^{ \pm} V_{j}$ acting in $L^{2}(\mathbb{R})$. Then the essential spectrum of $H$ is the union of the spectra of the four asymptotic Hamiltonians. The eigenvalues of the internal Hamiltonians and the numbers $c_{1}^{+} c_{2}^{+}, c_{1}^{+} c_{2}^{-}, c_{1}^{-} c_{2}^{+}, c_{1}^{-} c_{2}^{-}$compose the set of thresholds. If the critical set $\kappa(H)$ is defined as the set of these thresholds and of the eigenvalues of $H$, we prove a Mourre estimate and deduce a limiting absorption principle on $\mathbb{R} \backslash \kappa(H)$, and thus get the absence of singularly continuous spectrum. For the scattering, let us make some heuristic discussion and get some physical intuition. Consider a state in the absolutely continuous subspace of $L^{2}\left(\mathbb{R}^{2}\right)$ with respect to $H$ propagating into the positive quadrant of $\mathbb{R}^{2}$. We can expect that its asymptotic evolution is governed by the operator $-\boldsymbol{\Delta}+c_{1}^{+} c_{2}^{+}$, and thus this state will be asymptotically free. But there might also exist some infinite valley parallel to one of the axis which could trap some scattering states. And such states would then behave asymptotically like guided waves.

This variety of possible outcomes for the asymptotic evolution is one of the reasons for the complexity of the analysis of anisotropic systems. In order to predict the asymptotic behaviour of a given scattering state, one has to know roughly its asymptotic localization. It seems to us that the right concept for obtaining this information is the asymptotic velocity. In the previous example, the asymptotic velocity of the asymptotically free state points out in the positive quadrant, while for the asymptotically guided state, the asymptotic velocity has a zero component. Such characteristics will be used for classifying the scattering states.

Let us briefly describe our mathematical tools. For the spectral analysis, we mainly use the method of the conjugate operator in the algebraic framework developed by W. O. Amrein, A. Boutet de Monvel and V. Georgescu [1]. In this approach, the main object of the theory is a $\mathrm{C}^{*}$-subalgebra $\mathfrak{C}$ of the set of bounded linear operators in some Hilbert space $\mathcal{H}$. This $\mathrm{C}^{*}$-algebra is closely related to the anisotropy. The operators $H$ under consideration are then self- 
adjoint operators in $\mathcal{H}$ affiliated to $\mathfrak{C}$, i.e. the resolvent $(H-z)^{-1}$ belongs to $\mathfrak{C}$ for any complex number $z$ with non-zero imaginary part. We also rely upon the recent idea that a class of functions defined on $\mathbb{R}^{n}$ having a certain type of anisotropy is associated with a compactification of $\mathbb{R}^{n}$, the one on which all these functions admit a continuous extension. We refer to [2], [8] and to [12], [13] of M. Măntoiu for motivations, for some general principles and in particular for the use of crossed products in relation with spectral analysis. For the scattering theory, the strategy of J. Dereziński and C. Gérard exposed in [4], Sections 6.6 and 6.7, is followed. Various propagation estimates are proved with the help of some propagation observables and with a partition of unity inspired by the paper of G. M. Graf [9]. The notions of minimal and maximal velocities are introduced and the asymptotic velocity is used for the definition of the wave operators and the proof of asymptotic completeness.

In the sequel we shall consider potentials $V$ such that $\lim _{x_{j} \rightarrow \pm \infty} V(\cdot)$ exist for each $j \in\{1, \ldots, n\}$ in a suitable sense, and call them cartesian potentials. This leads to a natural $n$-dimensional generalization of certain situations considered in [3] and [7]. The underlying compactification of $\mathbb{R}^{n}$ is the cartesian product of $n$ copies of the two-point compactification $\overline{\mathbb{R}}:=\{-\infty\} \sqcup \mathbb{R} \sqcup\{+\infty\}$ of $\mathbb{R}$. Hence let us define $\overline{\mathbb{R}}^{n}:=\overline{\mathbb{R}}_{1} \times \cdots \times \overline{\mathbb{R}}_{n}$ (the indexation corresponds to that of the variables) endowed with the product topology, and let $C\left(\overline{\mathbb{R}}^{n}\right)$ denote the algebra of continuous complex functions on $\overline{\mathbb{R}}^{n}$. This algebra is naturally identified with a subalgebra of $B C_{u}\left(\mathbb{R}^{n}\right)$, the bounded uniformly continuous complex functions on $\mathbb{R}^{n}$. The precise definition of cartesian potentials is given in Definition 4.1. However, let us already mention that any real element of $C\left(\overline{\mathbb{R}}^{n}\right)$ is a smooth cartesian potential.

We introduce some notations which are needed for the statement of our results. Let $\mathcal{L}$ be the set of all multi-indexes $\alpha=\left\{\alpha_{j}\right\}_{j=1}^{n}$ with $\alpha_{j}$ taking values in $\{-1,0,1\}$. There exists a one-to-one relation between $\mathcal{L}$ and all generalized hypersurfaces of an $n$-dimensional hypercube. Indeed, the hypersurface $\overline{\mathbb{R}}^{\alpha}:=$ $\overline{\mathbb{R}}_{1}^{\alpha_{1}} \times \cdots \times \overline{\mathbb{R}}_{n}^{\alpha_{n}}$ (with the convention that $\overline{\mathbb{R}}_{j}^{0}=\overline{\mathbb{R}}_{j}$ and $\overline{\mathbb{R}}_{j}^{ \pm 1}=\left\{ \pm \infty_{j}\right\}$ ) is a generalized face of $\overline{\mathbb{R}}^{n}$. Endowed with the induced topology, its interior is clearly isomorphic to $\mathbb{R}^{\alpha}:=\prod_{\left\{j \mid \alpha_{j}=0\right\}} \mathbb{R}_{j}$ or to $\{0\}$. We symbolize by $|\alpha|$ the dimension of the vector space $\mathbb{R}^{\alpha}$. For $|\alpha| \neq 0$, let $\mathcal{H}_{\alpha}$ denote the Hilbert space $L^{2}\left(\mathbb{R}^{\alpha}\right)$ and let $\mathcal{H}_{\alpha}^{2}$ be the usual Sobolev space of order two on $\mathbb{R}^{\alpha}$. This space is the domain of the Laplace operator $\boldsymbol{\Delta}^{\alpha}:=\sum_{\left\{j \mid \alpha_{j}=0\right\}} \boldsymbol{\Delta}_{j}$. If $\alpha=o:=(0, \ldots, 0)$ the familiar notations are kept: $\mathbb{R}^{o}=\mathbb{R}^{n}, \mathcal{H}_{o}=\mathcal{H}, \mathcal{H}_{o}^{2}=\mathcal{H}^{2}$ and $\boldsymbol{\Delta}^{o}=\boldsymbol{\Delta}$. In the special cases $|\alpha|=0$, meaning that $\overline{\mathbb{R}}^{\alpha}$ is a corner of the hypercube, we take by convention $\mathcal{H}_{\alpha}=\mathcal{H}_{\alpha}^{2}=\mathbb{C}$. 
For any function $V \in C\left(\overline{\mathbb{R}}^{n}\right)$, its restriction $V^{\alpha}$ to the hypersurface $\overline{\mathbb{R}}^{\alpha}$ is identified with an element of $B C_{u}\left(\mathbb{R}^{\alpha}\right)$. One notices that the expression $V^{\alpha}(x)$ with $x \in \mathbb{R}^{n}$ has an unambiguous meaning. Indeed, the algebra $B C_{u}\left(\mathbb{R}^{\alpha}\right)$ is canonically identified with a subalgebra of $B C_{u}\left(\mathbb{R}^{n}\right)$, its elements depending only on the variables $x_{j}$ for which $\alpha_{j}=0$. More generally, for any cartesian potential $V$ the restriction $V^{\alpha}$ of $V$ to the hypersurface $\overline{\mathbb{R}}^{\alpha}$ also exists in a generalized sense (cf. Definition 4.1). Thus we may set $H_{\alpha}:=-\boldsymbol{\Delta}+V^{\alpha}$ and $H^{\alpha}:=-\boldsymbol{\Delta}^{\alpha}+V^{\alpha}$, the former being a self-adjoint operator in $\mathcal{H}$ with domain $\mathcal{H}^{2}$ and the latter a self-adjoint operator in $\mathcal{H}_{\alpha}$ with domain $\mathcal{H}_{\alpha}^{2}$. Let $\sigma_{p}(\cdot)$ denote the point spectrum of any self-adjoint operator. With the cartesian Hamiltonian $H \equiv H^{o}=-\boldsymbol{\Delta}+V$, one associates two special sets: the set of thresholds $\tau(H)=\cup_{\alpha \neq o} \sigma_{p}\left(H^{\alpha}\right)$, and $\kappa(H)=\cup_{\alpha \in \mathcal{L}} \sigma_{p}\left(H^{\alpha}\right)$, the critical set of $H$.

In order to give a precise description of the spectrum $\sigma(H)$ of $H$, some regularity of the potential with respect to the generator $A$ of dilations has to be imposed. We refer to Section 2 for the description of this regularity (including the definition of the class $\left.C^{1,1}(A)\right)$ and to Section 5 for its compatibility with the cartesian anisotropy. If $\mathcal{G}$ is a Banach space, its norm is written $\|\cdot\|_{\mathcal{G}}$. The weighted Sobolev space $\mathcal{H}_{t}^{s}$ is the closure of the Schwartz space on $\mathbb{R}^{n}$ with respect to the norm $\|\cdot\|_{\mathcal{H}_{t}^{s}}=\left\|\left(1+P^{2}\right)^{s / 2}\left(1+Q^{2}\right)^{t / 2} \cdot\right\|$, where $P_{j}:=-i \nabla_{j}$, $j \in\{1, \ldots, n\}$, are the components of the momentum operator and $Q_{j}$ is the operator of multiplication by the variable $x_{j}$. If $t=0$, we simply omit this index.

Theorem 1.1. Let $H=-\boldsymbol{\Delta}+V$ with $V$ a cartesian potential. Then

i) $\sigma_{\text {ess }}(H)=\left[\min _{|\alpha|=n-1} \inf \sigma\left(H^{\alpha}\right), \infty\right)$.

Furthermore, if $V$ is of class $C^{1,1}(A)$, with $A$ the generator of dilations, then

ii) $\tau(H)$ and $\kappa(H)$ are closed countable sets, the eigenvalues of $H$ not belonging to $\tau(H)$ are of finite multiplicity and can accumulate only at points of $\tau(H)$,

iii) H has no singularly continuous spectrum,

iv) for each $\delta>0$, there exists $c<\infty$ such that $\left|\left\langle\varphi,(H-\lambda \pm i \mu)^{-1} \varphi\right\rangle\right| \leq$ $c\|\varphi\|_{\mathcal{H}_{1 / 2+\delta}^{-1}}^{2}$ for all $\varphi \in \mathcal{H}_{1 / 2+\delta}^{-1}$ and uniformly in $\lambda$ on each compact subset of $\mathbb{R} \backslash \kappa(H)$ and in $\mu>0$.

We mention that there exists a slightly stronger version of the limiting absorption principle in terms of Besov spaces [1]. For reasons of simplicity we do not take this improvement into account. 
Let us recall that the asymptotic velocity $\mathcal{P}$ for a system described by $H$ is

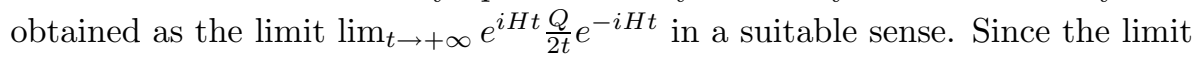
$t \rightarrow-\infty$ is completely similar, we do not consider it. We denote by $\mathcal{P}_{\alpha}$ the asymptotic velocity obtained for $H_{\alpha}$. The following partition of $\mathbb{R}^{n}$ is useful for the description of the different possible outcomes of the asymptotic evolution. For each $\alpha \in \mathcal{L}$, we define

$$
Z_{\alpha}:=\left\{x \in \mathbb{R}^{n} \mid x_{j}=0 \text { if } \alpha_{j}=0 \text { and } \alpha_{j} x_{j}>0 \text { if } \alpha_{j} \neq 0\right\} .
$$

We shall prove that for $\alpha \neq o$, the elements of $\mathcal{H}$ with support of their asymptotic velocity on $Z_{\alpha}$ have an asymptotic evolution governed by the Hamiltonian $H_{\alpha}$. For this purpose, we roughly impose that the potential $V$ approaches its limits at infinity in a short-range way. A more precise condition is given in Section 7 , equation (20).

If $C$ is an $m$-tuple of commuting self-adjoint operators ( $m$ a positive integer), we denote by $E_{\Xi}(C)$ its spectral projection corresponding to the subset $\Xi \subset \mathbb{R}^{m}$. We also use the notation $E_{\mathrm{p}}(B)$ for the orthogonal projection on the subspace spanned by the eigenvectors of a self-adjoint operator $B$.

Theorem 1.2. $\quad$ Let $V$ be a cartesian potential of class $C^{1,1}(A)$ satisfying (20), with $A$ the generator of dilations. Then for each $\alpha \in \mathcal{L}$,

i) the operator $\Omega_{\alpha}^{+}:=s-\lim _{t \rightarrow+\infty} e^{i H t} e^{-i H_{\alpha} t} E_{z_{\alpha}}\left(\mathcal{P}_{\alpha}\right)$ exists, and its range $\operatorname{Ran} \Omega_{\alpha}^{+}$is equal to $E_{z_{\alpha}}(\mathcal{P}) \mathcal{H}$,

ii) if $\beta \neq \alpha$, then $\operatorname{Ran} \Omega_{\beta}^{+}$is orthogonal to $\operatorname{Ran} \Omega_{\alpha}^{+}$; furthermore the direct sum $\bigoplus_{\beta \neq o} \operatorname{Ran} \Omega_{\beta}^{+}$spans the absolutely continuous subspace of $\mathcal{H}$ with respect to $H$,

iii) if $\mathcal{H}$ is identified with $\left(\bigotimes_{\left\{j \mid \alpha_{j} \neq 0\right\}} L^{2}\left(\mathbb{R}_{j}\right)\right) \otimes \mathcal{H}_{\alpha}$, the spectral projection $E_{z_{\alpha}}\left(\mathcal{P}_{\alpha}\right)$ is equal to $\left(\bigotimes_{\left\{j \mid \alpha_{j} \neq 0\right\}} E_{\left\{y \in \mathbb{R} \mid \alpha_{j} y>0\right\}}\left(P_{j}\right)\right) \otimes E_{\mathrm{p}}\left(H^{\alpha}\right)$.

Let us notice that the projections $E_{z_{\alpha}}(\mathcal{P})$ correspond to the projections $P^{+}(E)$ conjectured in the Introduction of [3]. In relation with this result, we mention the recent work of Y. Dermenjian and V. Iftimie in the case of perturbed stratified media [5]. Their results are comparable but the anisotropy they consider is less general than ours since it is a short-range perturbation of a $L^{\infty}$-function which depends only on the variable $x_{n}$ and admits limits as $x_{n} \rightarrow \pm \infty$. 
In Section 2 we describe the algebraic framework and some generalities on the regularity of $H$ with respect to the conjugate operator. The algebra related to the cartesian anisotropy is introduced in Section 3, where its rich internal structure is investigated. It already gives some informations on the essential spectrum. In order to deal with non-smooth potentials, some technicalities are needed. Section 4 is devoted to this purpose. Definition 4.1 contains the description of a generalized class of cartesian potentials, which includes $C\left(\overline{\mathbb{R}}^{n}\right)$. The affiliation of the corresponding cartesian Hamiltonians to the mentioned algebra is proved. The Mourre estimate and the limiting absorption principle are elaborated in Section 5, where the proof of Theorem 1.1 is given. The last two sections are dedicated to the scattering theory. Section 6 deals with the asymptotic velocity $\mathcal{P}$ and some of its properties. In Section 7 , we use it to construct the wave operators and to prove Theorem 1.2.

We end the Introduction with two observations. The first one concerns the relationship between cartesian and N-body Hamiltonians. Although our approach for the spectral and scattering theory of the former is similar to that developed for the latter, potentials which are both cartesian and of N-body type are very special cases of cartesian potentials and of N-body potentials. Indeed, in the formalism of generalized N-body systems (see Section 5.1 of [4]) such potentials correspond to a system related to a finite semilattice of subspaces of $\mathbb{R}^{n}$ which satisfy some orthogonality relations; on the other hand, as cartesian potentials, they must converge to zero (in a suitable sense) except in the vicinity of some subspaces of $\mathbb{R}^{n}$ of lower dimensions. The second observation is that the difficulties due to the anisotropy already appear in two dimensions, a situation which is easily visualized. Therefore this model is, undoubtedly, of pedagogical interest. For convenience, we have included some relevant examples of cartesian potentials in Sections 4, 5 and 7 .

\section{$\S 2 . \quad$ The Algebraic Framework}

Let us consider a self-adjoint operator $H$ in a Hilbert space $\mathcal{H}$. The spectrum and the essential spectrum of $H$ can be expressed in terms of its continuous functional calculus: ${ }^{1}$

$$
\begin{aligned}
\sigma(H) & =\left\{\lambda \in \mathbb{R} \mid \text { if } \eta \in C_{0}(\mathbb{R}) \text { and } \eta(\lambda) \neq 0, \text { then } \eta(H) \neq 0\right\}, \\
\sigma_{\text {ess }}(H) & =\left\{\lambda \in \mathbb{R} \mid \text { if } \eta \in C_{0}(\mathbb{R}) \text { and } \eta(\lambda) \neq 0, \text { then } \eta(H) \notin \mathcal{K}(\mathcal{H})\right\} .
\end{aligned}
$$

\footnotetext{
${ }^{1}$ If $m$ and $k$ are positive integers, we denote by $C_{0}\left(\mathbb{R}^{m}\right)$ the set of all continuous complex functions on $\mathbb{R}^{m}$ converging to zero at infinity, and by $C_{c}^{k}\left(\mathbb{R}^{m}\right)$ the subset of $C_{0}\left(\mathbb{R}^{m}\right)$ of $k$ times continuously differentiable functions of compact support. For any Hilbert spaces $\mathcal{H}$ and $\mathcal{G}, \mathcal{B}(\mathcal{H}, \mathcal{G})$ denotes the Banach space of bounded linear operators from $\mathcal{H}$ to $\mathcal{G}$, $\mathcal{B}(\mathcal{H}):=\mathcal{B}(\mathcal{H}, \mathcal{H})$ and $\mathcal{K}(\mathcal{H})$ is the ideal of compact operators in $\mathcal{H}$.
} 
If $\mathfrak{C}$ is a $\mathrm{C}^{*}$-subalgebra of $\mathcal{B}(\mathcal{H})$, then $H$ is said to be affiliated to $\mathfrak{C}$ if $\eta(H) \in \mathfrak{C}$ for all $\eta \in C_{0}(\mathbb{R})$. A sufficient condition is that $(H-z)^{-1} \in \mathfrak{C}$ for some $z \in \mathbb{C} \backslash \sigma(H)$.

The above situation is a special case of the following more abstract framework:

\section{Definition 2.1.}

i) A self-adjoint observable affiliated to a $\mathrm{C}^{*}$-algebra $\mathfrak{C}$ is a functional calculus taking value in $\mathfrak{C}$, i.e. a ${ }^{*}$-morphism $H: C_{0}(\mathbb{R}) \rightarrow \mathfrak{C}$. The notation $\eta(H)$ will be used instead of $H(\eta)$.

ii) The spectrum $\sigma(H)$ of the observable $H$ is the set of real values $\lambda$ such that, whenever $\eta \in C_{0}(\mathbb{R})$ and $\eta(\lambda) \neq 0$, then $\eta(H) \neq 0$.

iii) If $\pi: \mathfrak{C} \rightarrow \mathfrak{C}^{\prime}$ is a ${ }^{*}$-morphism between two $\mathrm{C}^{*}$-algebras and $H$ is a selfadjoint observable affiliated to $\mathfrak{C}$, then $\pi(H): C_{0}(\mathbb{R}) \rightarrow \mathfrak{C}^{\prime}$, given by $\eta(\pi(H)):=\pi(\eta(H))$, is a self-adjoint observable affiliated to $\mathfrak{C}^{\prime}$. We call it the image of $H$ through $\pi$.

In the sequel we shall simply write morphism for *-morphism between two $\mathrm{C}^{*}$-algebras.

We recall some definitions related to the Mourre estimate and refer to [1] for details and a self-contained presentation. Let $\left\{W_{t}\right\}_{t \in \mathbb{R}}$ be the unitary group in $\mathcal{H}$ generated by a self-adjoint operator $A$. For any $B \in \mathcal{B}(\mathcal{H})$, we write $B \in C^{1}(A)$ if the mapping $\mathbb{R} \ni t \mapsto W_{-t} B W_{t} \in \mathcal{B}(\mathcal{H})$ is strongly $C^{1}$. If this mapping is $C^{1}$ in norm we write $B \in C_{u}^{1}(A)$. By assuming that $B \in C^{1}(A)$, we give a rigorous sense to the commutator $[B, i A] \in \mathcal{B}(\mathcal{H})$.

A self-adjoint operator $H$ in $\mathcal{H}$ is of class $C^{1}(A)$ (resp. $\left.C_{u}^{1}(A)\right)$ if $(H-$ $z)^{-1} \in C^{1}(A)$ (resp. $\left.(H-z)^{-1} \in C_{u}^{1}(A)\right)$ for some, and then for all, $z \in$ $\mathbb{C} \backslash \sigma(H)$. Let $\mathcal{G}$ be the domain of $H$ endowed with the graph norm and assume that it is left invariant by the group $\left\{W_{t}\right\}_{t \in \mathbb{R}}$. We denote by $\mathcal{G}^{*}$ its dual space and by $\left\{W_{t}^{*}\right\}_{t \in \mathbb{R}}$ the standard $\mathrm{C}_{0}$-group obtained by duality from the action of the group restricted to $\mathcal{G}$. Then $H$ is of class $C^{1}(A)$ if and only if the mapping $\mathbb{R} \ni t \mapsto W_{t}^{*} H W_{t} \in \mathcal{B}\left(\mathcal{G}, \mathcal{G}^{*}\right)$ is strongly $C^{1}$ (see [1], Theorem 6.3.4). In this case, the commutator $[H, i A]$ belongs unambigously to $\mathcal{B}\left(\mathcal{G}, \mathcal{G}^{*}\right)$. 
With any $H$ of class $C^{1}(A)$, one associates the functions $\varrho_{H}^{A}$ and $\varrho_{H}^{A}$ defined on $\mathbb{R}$ with values in $(-\infty, \infty]$ by

$$
\begin{aligned}
\varrho_{H}^{A}(\lambda)=\sup \{a \in \mathbb{R} \mid & \exists \eta \in C_{c}^{\infty}(\mathbb{R}) \text { s.t. } \eta(\lambda) \neq 0 \text { and } \\
& \left.a \eta^{2}(H) \leq \eta(H)[H, i A] \eta(H)\right\}, \\
\tilde{\varrho}_{H}^{A}(\lambda)=\sup \{a \in \mathbb{R} \mid & \exists \eta \in C_{c}^{\infty}(\mathbb{R}) \text { and } K \in \mathcal{K}(\mathcal{H}) \text { s.t. } \eta(\lambda) \neq 0 \\
& \text { and } \left.a \eta^{2}(H)+K \leq \eta(H)[H, i A] \eta(H)\right\} .
\end{aligned}
$$

Some properties of these functions will be quoted later on (Proposition 5.1). The Mourre set of $H$ with respect to $A$ is $\mu^{A}(H):=\left\{\lambda \in \mathbb{R} \mid \varrho_{H}^{A}(\lambda)>0\right\}$. Since the work of Mourre $([14],[15])$, it is known that $H$ has nice spectral properties on this set. In particular $H$ has no eigenvalue in $\mu^{A}(H)$ and, under an additional regularity assumption, a limiting absorption principle can be stated on it. This additional condition is as follows: for some, and then for all, $z \in \mathbb{C} \backslash \sigma(H), \int_{0}^{1}\left\|W_{-t}(H-z)^{-1} W_{t}+W_{t}(H-z)^{-1} W_{-t}-2(H-z)^{-1}\right\| \frac{d t}{t^{2}}<\infty$. If this condition is satisfied, $H$ is said to be of class $C^{1,1}(A)$. Assuming the invariance of $\mathcal{G}$ under each $W_{t}$, an equivalent requirement (see Theorem 6.3.4 of [1]) is that $\int_{0}^{1}\left\|W_{t}^{*} H W_{t}+W_{-t}^{*} H W_{-t}-2 H\right\|_{\mathcal{G} \rightarrow \mathcal{G}^{*}} \frac{d t}{t^{2}}<\infty$, where $\|\cdot\|_{\mathcal{G} \rightarrow \mathcal{G}^{*}}$ is the norm of $\mathcal{B}\left(\mathcal{G}, \mathcal{G}^{*}\right)$.

In our applications, $H$ is equal to $-\boldsymbol{\Delta}+V$ in $\mathcal{H}=L^{2}\left(\mathbb{R}^{n}\right)$ with domain $\mathcal{H}^{2}$, the Sobolev space of order two on $\mathbb{R}^{n}$. The unitary group $\left\{W_{t}\right\}_{t \in \mathbb{R}}$ is the group of dilations, which leaves $\mathcal{H}^{2}$ invariant. Since $W_{t}^{*} \boldsymbol{\Delta} W_{t}=e^{2 t} \boldsymbol{\Delta}$, an easy calculation shows that the operator $-\boldsymbol{\Delta}$ satisfies the $C^{1,1}(A)$-condition. Hence $H$ is of class $C^{1,1}(A)$ if $V$ is $\Delta$-bounded with relative bound less than one and is of class $C^{1,1}(A)$. We still recall some definitions related to this condition in such a setting.

Definition 2.2. Let $U: \mathcal{H}^{2} \rightarrow \mathcal{H}$ be a linear symmetric operator.

i) We say that $U$ is a Mourre potential if the sesquilinear form $[[U, A], A]$ defined on the Schwartz space on $\mathbb{R}^{n}$ is continuous for the topology induced by $\mathcal{H}^{2}$.

ii) We say that $U$ is a long-range potential if $[U, A] \in \mathcal{B}\left(\mathcal{H}^{2}, \mathcal{H}^{-1}\right)$ and if there exists a function $\xi \in C^{\infty}\left(\mathbb{R}^{n}\right)$ with $\xi(x)=0$ if $|x| \leq 1$ and $\xi(x)=1$ if $|x| \geq 2$ such that

$$
\int_{1}^{\infty}\left\|\xi\left(\frac{Q}{r}\right)[U, A]\right\|_{\mathcal{H}^{2} \rightarrow \mathcal{H}^{-1}} \frac{d r}{r}<\infty
$$

iii) We say that $U$ is a short-range potential if $\int_{1}^{\infty}\left\|\xi\left(\frac{Q}{r}\right) U\right\|_{\mathcal{H}^{2} \rightarrow \mathcal{H}} d r<\infty$ for some $\xi \in C^{\infty}\left(\mathbb{R}^{n}\right)$ such that $\xi(x)=0$ if $|x| \leq 1$ and $\xi(x)=1$ if $|x| \geq 2$. 
It is shown in [1] that in all three cases, $U$ is of class $C^{1,1}(A)$. These definitions are useful in order to construct examples of cartesian potentials of this class. We shall make some remarks on this point at the end of Section 5 .

\section{$\S 3 . \quad$ The Cartesian Algebra and the Essential Spectrum}

In this section, we study the cartesian algebra $\mathfrak{C}$ which characterizes in some sense the Hamiltonians under consideration. Its properties will be extensively used in our later proofs. Let us first observe that $\mathcal{L}$ is naturally endowed with the structure of a finite semilattice, with largest element $o: \beta \leq \alpha$ if $\overline{\mathbb{R}}^{\beta} \subset \overline{\mathbb{R}}^{\alpha}$. $\beta<\alpha$ means strict ordering, and we write $\beta \lessdot \alpha$ if $\beta<\alpha$ and $\overline{\mathbb{R}}^{\beta} \subset \overline{\mathbb{R}}^{\gamma} \subset \overline{\mathbb{R}}^{\alpha}$ implies that either $\gamma=\beta$ or $\gamma=\alpha$. For $j \in\{1, \ldots, n\}$, let $(\beta-\alpha)_{j}$ be equal to $\beta_{j}-\alpha_{j}$. One has equivalently that $\beta \leq \alpha$ if, whenever $\alpha_{j} \neq 0$, then $\beta_{j}=\alpha_{j}$, and that $\beta \lessdot \alpha$ if and only if $\beta \leq \alpha$ and there is exactly one value of $j$ such that $(\beta-\alpha)_{j} \neq 0$. One also notices that $|\alpha|$ is equal to $n-\sum_{j=1}^{n}\left|\alpha_{j}\right|$.

In the sequel, we shall make some abuses of notation: $\overline{\mathbb{R}}^{\alpha}$ will denote either a hypersurface of $\overline{\mathbb{R}}^{n}$ or the isomorphic cartesian product of $\overline{\mathbb{R}}_{j}$ for all $\alpha_{j}=0$ (a $|\alpha|$-dimensional hypercube). Similarly, $C\left(\overline{\mathbb{R}}^{\alpha}\right)$ will be viewed either as a $\mathrm{C}^{*}$-algebra on its own, or as a subalgebra of $C\left(\overline{\mathbb{R}}^{n}\right)$ with elements depending only on the variables $x_{j}$ for which $\alpha_{j}=0$. However, in every case, the context should suppress the ambiguity.

Before defining $\mathfrak{C}$, we summarize some easy properties of the abelian algebra $C\left(\overline{\mathbb{R}}^{n}\right)$. For each $\alpha \in \mathcal{L}$, let us show the invariance of the hypersurface $\overline{\mathbb{R}}^{\alpha}$ under the natural action $\mathcal{U}^{o}$ of $\mathbb{R}^{n}$ on $\overline{\mathbb{R}}^{n}$ by translations. For $y \in \mathbb{R}$, let $U_{y}: \overline{\mathbb{R}} \rightarrow \overline{\mathbb{R}}$ with $U_{y}(z)=z+y$ if $z \in \mathbb{R}$ and $U_{y}( \pm \infty)= \pm \infty$, be the extension to $\overline{\mathbb{R}}$ of the translation by $y$ on $\mathbb{R}$. Since $\overline{\mathbb{R}}^{n}$ equals $\overline{\mathbb{R}}_{1} \times \cdots \times \overline{\mathbb{R}}_{n}$, the action of the group on $\overline{\mathbb{R}}^{n}$ can be defined componentwise: $\left[\mathcal{U}_{x}^{o}(z)\right]_{j}=U_{x_{j}}\left(z_{j}\right)$ for any $z \in \overline{\mathbb{R}}^{n}$ and $x \in \mathbb{R}^{n}$. But $\{-\infty\},\{+\infty\}$ and $\overline{\mathbb{R}}$ are invariant under each homeomorphism $U_{y}$, and therefore $\overline{\mathbb{R}}^{\alpha}$ is invariant. Consequently, each subalgebra $C\left(\overline{\mathbb{R}}^{\alpha}\right)$ of $C\left(\overline{\mathbb{R}}^{n}\right)$ is stable under the action of translations. Indeed, the group $\mathcal{U}^{\circ}$ of homeomorphisms induces a representation of the translation group by ${ }^{*}$-automorphisms of $C\left(\overline{\mathbb{R}}^{\alpha}\right)$ : for $f \in C\left(\overline{\mathbb{R}}^{\alpha}\right), x \in \mathbb{R}^{n}$ and $z \in \overline{\mathbb{R}}^{\alpha}$, $\left(\mathcal{U}_{x}^{o}(f)\right)(z)=f\left(\mathcal{U}_{x}^{o}(z)\right)$. In particular, it implies the stability of $C\left(\overline{\mathbb{R}}^{n}\right)$ under $\mathcal{U}^{o}$, and similarly the stability of the $\mathrm{C}^{*}$-algebra $C\left(\overline{\mathbb{R}}^{\alpha}\right)$ under $\mathcal{U}^{\alpha}$, where $\mathcal{U}^{\alpha}$ is the corresponding action of $\mathbb{R}^{\alpha}$ on $\overline{\mathbb{R}}^{\alpha}$.

For each subalgebra $C\left(\overline{\mathbb{R}}^{\alpha}\right)$ of $C\left(\overline{\mathbb{R}}^{n}\right)$, there exists a morphism $\pi_{\alpha}$ : $C\left(\overline{\mathbb{R}}^{n}\right) \ni f \mapsto \pi_{\alpha}(f) \equiv f^{\alpha} \in C\left(\overline{\mathbb{R}}^{\alpha}\right)$ given by restriction of $f$ to the hypersurface $\overline{\mathbb{R}}^{\alpha}$. This morphism is covariant since the relation $\pi_{\alpha} \circ \mathcal{U}_{x}^{o}=\mathcal{U}_{x}^{o} \circ \pi_{\alpha}$ 
is satisfied for all $x \in \mathbb{R}^{n}$. Let $C_{0}\left(\mathbb{R}^{n}\right)$ be identified with the ideal of functions in $C\left(\overline{\mathbb{R}}^{n}\right)$ which are null on the boundary $\overline{\mathbb{R}}^{n} \backslash \mathbb{R}^{n}$. A certain direct sum of morphisms $\pi_{\alpha}$ has an important feature: $\oplus_{\alpha \lessdot o} \pi_{\alpha}: C\left(\overline{\mathbb{R}}^{n}\right) \rightarrow \oplus_{\alpha \lessdot o} C\left(\overline{\mathbb{R}}^{\alpha}\right)$ is a covariant morphism with kernel equal to $C_{0}\left(\mathbb{R}^{n}\right)$. Thus there exists a natural injective morphism

$$
\pi: C\left(\overline{\mathbb{R}}^{n}\right) / C_{0}\left(\mathbb{R}^{n}\right) \hookrightarrow \oplus_{\alpha \lessdot o} C\left(\overline{\mathbb{R}}^{\alpha}\right) .
$$

We now identify $C\left(\overline{\mathbb{R}}^{\alpha}\right)$ with the subalgebra of $\mathcal{B}\left(\mathcal{H}_{\alpha}\right)$ consisting of all multiplication operators $f(Q)$ with $f \in C\left(\overline{\mathbb{R}}^{\alpha}\right)$. $C_{0}\left(\mathbb{R}^{\alpha *}\right)$ denotes the set of operators $h(P):=\mathcal{F}_{\alpha}^{*} h(Q) \mathcal{F}_{\alpha}$ with $h \in C_{0}\left(\mathbb{R}^{\alpha}\right)$ and where $\mathcal{F}_{\alpha}$ is the Fourier transform in $\mathcal{H}_{\alpha}$ (we have identified the dual of $\mathbb{R}^{\alpha}$ with $\mathbb{R}^{\alpha}$ itself). A few elements from the theory of crossed products are used in the sequel. We refer to [8], Sections 3 and 4 for an overview on this subject in relation with spectral analysis. This reference includes some precise definitions and all the required results.

One defines $\mathfrak{C}^{\alpha}:=\left\langle C\left(\overline{\mathbb{R}}^{\alpha}\right) \cdot C_{0}\left(\mathbb{R}^{\alpha *}\right)\right\rangle$, the norm closure in $\mathcal{B}\left(\mathcal{H}_{\alpha}\right)$ of the set of finite sums of the form $f_{1}(Q) h_{1}(P)+\cdots+f_{N}(Q) h_{N}(P)$ with $f_{k} \in C\left(\overline{\mathbb{R}}^{\alpha}\right)$ and $h_{k} \in C_{0}\left(\mathbb{R}^{\alpha}\right)$. It is shown in [8], Theorem 4.1, that $\mathfrak{C}^{\alpha}$ is a $\mathrm{C}^{*}$-algebra; the stability of $C\left(\overline{\mathbb{R}}^{\alpha}\right)$ under $\mathcal{U}^{\alpha}$ is here essential. Moreover, this algebra is isomorphic to the crossed product $C\left(\overline{\mathbb{R}}^{\alpha}\right) \rtimes \mathbb{R}^{\alpha}$, which is defined abstractly in terms of the action of translations on $C\left(\overline{\mathbb{R}}^{\alpha}\right)$. In the special case $\alpha=o$, we simply set $\mathfrak{C}:=\mathfrak{C}^{o}$. We shall give in Lemma 4.1 another description of this $\mathrm{C}^{*}$-algebra in terms of suitable limits at infinity. We also mention the following known relations:

$$
\mathcal{K}(\mathcal{H})=\left\langle C_{0}\left(\mathbb{R}^{n}\right) \cdot C_{0}\left(\mathbb{R}^{n *}\right)\right\rangle \cong C_{0}\left(\mathbb{R}^{n}\right) \rtimes \mathbb{R}^{n} .
$$

Due to the embedding of $C\left(\overline{\mathbb{R}}^{\alpha}\right)$ into $C\left(\overline{\mathbb{R}}^{n}\right)$ and its stability under $\mathcal{U}^{o}$, one may form $\left\langle C\left(\overline{\mathbb{R}}^{\alpha}\right) \cdot C_{0}\left(\mathbb{R}^{n *}\right)\right\rangle$, which is a $C^{*}$-subalgebra of $\mathfrak{C}$ isomorphic to $C\left(\overline{\mathbb{R}}^{\alpha}\right) \rtimes \mathbb{R}^{n}$. Let $\mathbb{R}^{\alpha^{\perp}}$ denote the orthogonal complement of $\mathbb{R}^{\alpha}$ in $\mathbb{R}^{n}$. Proposition 2.4 of [19] asserts that $C\left(\overline{\mathbb{R}}^{\alpha}\right) \rtimes \mathbb{R}^{n}$ is isomorphic to $\left[\mathbb{C} \rtimes \mathbb{R}^{\alpha^{\perp}}\right] \otimes$ $\left[C\left(\overline{\mathbb{R}}^{\alpha}\right) \rtimes \mathbb{R}^{\alpha}\right]$. Hence, if $\mathcal{H}$ is identified with $L^{2}\left(\mathbb{R}^{\alpha^{\perp}}\right) \otimes \mathcal{H}_{\alpha}$, the $\mathrm{C}^{*}$-algebra $\left\langle C\left(\overline{\mathbb{R}}^{\alpha}\right) \cdot C_{0}\left(\mathbb{R}^{n *}\right)\right\rangle$ of bounded operators in $\mathcal{H}$ is isomorphic to the $\mathrm{C}^{*}$-algebra $C_{0}\left(\mathbb{R}^{\alpha^{\perp} *}\right) \otimes \mathfrak{C}^{\alpha}$ of bounded operators in $L^{2}\left(\mathbb{R}^{\alpha^{\perp}}\right) \otimes \mathcal{H}_{\alpha}$.

Since the morphism $\pi_{\alpha}$ is covariant, there exists a unique morphism

$$
\Pi_{\alpha}:\left\langle C\left(\overline{\mathbb{R}}^{n}\right) \cdot C_{0}\left(\mathbb{R}^{n *}\right)\right\rangle \rightarrow\left\langle C\left(\overline{\mathbb{R}}^{\alpha}\right) \cdot C_{0}\left(\mathbb{R}^{n *}\right)\right\rangle
$$

such that $\Pi_{\alpha}[f(Q) h(P)]=f^{\alpha}(Q) h(P)$ for each $f \in C\left(\overline{\mathbb{R}}^{n}\right)$ and each $h \in$ $C_{0}\left(\mathbb{R}^{n}\right)$. Furthermore, since $C_{0}\left(\mathbb{R}^{n}\right)$ is a stable ideal of $C\left(\overline{\mathbb{R}}^{n}\right)$, the general 
theory of crossed products gives the canonical isomorphism:

$$
\left[C\left(\overline{\mathbb{R}}^{n}\right) \rtimes \mathbb{R}^{n}\right] /\left[C_{0}\left(\mathbb{R}^{n}\right) \rtimes \mathbb{R}^{n}\right] \cong\left[C\left(\overline{\mathbb{R}}^{n}\right) / C_{0}\left(\mathbb{R}^{n}\right)\right] \rtimes \mathbb{R}^{n} .
$$

Using (1), (2) and some isomorphisms introduced above, one obtains:

$$
\begin{aligned}
\mathfrak{C} / \mathcal{K}(\mathcal{H}) & \cong\left[C\left(\overline{\mathbb{R}}^{n}\right) / C_{0}\left(\mathbb{R}^{n}\right)\right] \rtimes \mathbb{R}^{n} \\
& \hookrightarrow\left[\oplus_{\alpha \lessdot o} C\left(\overline{\mathbb{R}}^{\alpha}\right)\right] \rtimes \mathbb{R}^{n} \cong \oplus_{\alpha \lessdot o}\left[C\left(\overline{\mathbb{R}}^{\alpha}\right) \rtimes \mathbb{R}^{n}\right] \\
& \cong \oplus_{\alpha \lessdot o}\left\langle C\left(\overline{\mathbb{R}}^{\alpha}\right) \cdot C_{0}\left(\mathbb{R}^{n *}\right)\right\rangle .
\end{aligned}
$$

The resulting injective morphism is denoted by $\Pi$. But if $\Theta$ is the canonical surjection $\mathfrak{C} \rightarrow \mathfrak{C} / \mathcal{K}(\mathcal{H})$, then $\Pi \circ \Theta=\oplus_{\alpha \lessdot o} \Pi_{\alpha}$. Assume now that $H$ is a self-adjoint observable affiliated to $\mathfrak{C}$. Then $\sigma_{\text {ess }}(H)$ is equal to $\sigma[\Theta(H)]$, where $\Theta(H)$ is the image of $H$ in the Calkin algebra. Since an injective morphism preserves the spectrum, we have:

$$
\sigma_{\mathrm{ess}}(H)=\sigma(\Pi(\Theta(H)))=\sigma\left(\oplus_{\alpha \lessdot o} \Pi_{\alpha}(H)\right)=\bigcup_{\alpha \lessdot o} \sigma\left(\Pi_{\alpha}(H)\right),
$$

the last equality being valid because the spectrum of an observable affiliated to a finite direct sum is the union of the spectra of its components. Let us mention that some similar results were already obtained in [12].

\section{§4. Cartesian Hamiltonians}

Schrödinger operators $-\boldsymbol{\Delta}+V$ in $L^{2}\left(\mathbb{R}^{n}\right)$ affiliated to the $\mathrm{C}^{*}$-algebra $\mathfrak{C}$ are called cartesian. If $V$ is a real element of $C\left(\overline{\mathbb{R}}^{n}\right)$, the corresponding Hamiltonian is cartesian. This is easily seen by using the Neumann series $(-\boldsymbol{\Delta}+V-z)^{-1}=\sum_{k=0}^{\infty}(-\boldsymbol{\Delta}-z)^{-1}\left[V(z+\boldsymbol{\Delta})^{-1}\right]^{k}$ which is norm convergent for $|\Im z|$ large enough. In order to deal with non-smooth potentials, several technical results have to be obtained. This section is entirely devoted to this question.

In the sequel, we shall often use some non-decreasing functions $\xi$ in $C^{\infty}(\mathbb{R})$ satisfying $\xi(y)=0$ if $y \leq 1$ and $\xi(y)=1$ if $y \geq 2$. For reasons that will become obvious already in the next lemma, we call them asymptotic localization functions. Let us say that a bounded operator $B$ is semi-compact if $\zeta(Q) B$ is compact for all $\zeta \in C_{0}\left(\mathbb{R}^{n}\right)$. We recall that for each $\alpha \lessdot o$, there exists exactly one $j$ such that $\alpha_{j} \neq 0$. Hence $\alpha \cdot Q$ means $\alpha_{j} Q_{j}$ and therefore $\xi(\alpha \cdot Q)$ is well-defined for any function $\xi$ on $\mathbb{R}$. We start with a new description of $\mathfrak{C}$. 


\section{Lemma 4.1.}

i) Each operator in $\mathfrak{C}$ is semi-compact.

ii) A semi-compact operator $B$ belongs to $\mathfrak{C}$ if and only if there exist an asymptotic localization function $\xi$ and a family $\left\{B_{\alpha}\right\}_{\alpha \lessdot o}$ such that $B_{\alpha} \in$ $\left\langle C\left(\overline{\mathbb{R}}^{\alpha}\right) \cdot C_{0}\left(\mathbb{R}^{n *}\right)\right\rangle$ and $\lim _{r \rightarrow \infty}\left\|\xi\left(\alpha \cdot \frac{Q}{r}\right)\left(B-B_{\alpha}\right)\right\|=0$. Moreover, each operator $B_{\alpha}$ is unique and equal to $\Pi_{\alpha}(B)$.

Proof. a) By using (2) one observes that the product $\zeta(Q)[f(Q) h(P)]$ belongs to $\mathcal{K}(\mathcal{H})$ for any $\zeta, h \in C_{0}\left(\mathbb{R}^{n}\right)$ and any $f \in C\left(\overline{\mathbb{R}}^{n}\right)$. Since $\mathfrak{C}$ is the norm closure of the vector space generated by products of the form $f(Q) h(P)$ and since $\mathcal{K}(\mathcal{H})$ is norm closed, $\zeta(Q) B$ is compact for any $\zeta \in C_{0}\left(\mathbb{R}^{n}\right)$ and any $B \in \mathfrak{C}$. This proves i).

b) We now check the "only if" part of ii). Consider $f \in C\left(\overline{\mathbb{R}}^{n}\right)$ and $\alpha \in \mathcal{L}$. Let us observe that $f=f^{\alpha}+\left(f-f^{\alpha}\right)$, where $f^{\alpha} \in C\left(\overline{\mathbb{R}}^{\alpha}\right)$ and $\left(f-f^{\alpha}\right)$ belongs to the ideal $J_{\alpha}$ of functions of $C\left(\overline{\mathbb{R}}^{n}\right)$ which are equal to zero on the hypersurface $\overline{\mathbb{R}}^{\alpha}$. So, one has $C\left(\overline{\mathbb{R}}^{n}\right)=C\left(\overline{\mathbb{R}}^{\alpha}\right)+J_{\alpha}$, and $J_{\alpha}$ is nothing but the kernel of the morphism $\pi_{\alpha}$ of the previous section. By Corollary 3.1 of [8], one gets $\mathfrak{C}=\left\langle C\left(\overline{\mathbb{R}}^{\alpha}\right) \cdot C_{0}\left(\mathbb{R}^{n *}\right)\right\rangle+\left\langle J_{\alpha} \cdot C_{0}\left(\mathbb{R}^{n *}\right)\right\rangle$, where $\left\langle J_{\alpha} \cdot C_{0}\left(\mathbb{R}^{n *}\right)\right\rangle$ is the kernel of the morphism $\Pi_{\alpha}$. Now for any $B \in \mathfrak{C}$, we set $B_{\alpha}:=\Pi_{\alpha}(B) \in\left\langle C\left(\overline{\mathbb{R}}^{\alpha}\right)\right.$. $\left.C_{0}\left(\mathbb{R}^{n *}\right)\right\rangle$; then $B-B_{\alpha}$ is an element of $\left\langle J_{\alpha} \cdot C_{0}\left(\mathbb{R}^{n *}\right)\right\rangle$. If $\alpha \lessdot o$, it is easy to see that for any asymptotic localization function $\xi,\left\{1-\xi\left(\alpha \cdot \frac{Q}{r}\right)\right\}_{r \geq 1}$ is an approximate unit for this ideal and thus $\lim _{r \rightarrow \infty}\left\|\xi\left(\alpha \cdot \frac{Q}{r}\right)\left(B-B_{\alpha}\right)\right\|=0$.

c) To prove the "if" part in ii), let us introduce a partition of unity adapted to the anisotropy. Set $\xi_{0}(y):=1-\xi(y)-\xi(-y)$ for $y \in \mathbb{R}$, and for $x \in \mathbb{R}^{n}$ set $\xi_{\alpha}(x):=\prod_{\left\{j \mid \alpha_{j} \neq 0\right\}} \xi\left(\alpha_{j} x_{j}\right) \prod_{\left\{k \mid \alpha_{k}=0\right\}} \xi_{0}\left(x_{k}\right)$. For $\varepsilon>0$, there exists $r^{\prime}>0$ such that for all $r \geq r^{\prime}$ and all $\alpha \lessdot o,\left\|\xi\left(\alpha \cdot \frac{Q}{r}\right)\left(B-B_{\alpha}\right)\right\|<\frac{\varepsilon}{2\left(3^{n}-1\right)}$. For each $\alpha \lessdot o$, there exists $N_{\alpha}<\infty, f_{k}^{\alpha} \in C\left(\overline{\mathbb{R}}^{\alpha}\right)$ and $h_{k}^{\alpha} \in C_{0}\left(\mathbb{R}^{n}\right)$ such that $\left\|B_{\alpha}-\sum_{k=1}^{N_{\alpha}} f_{k}^{\alpha}(Q) h_{k}^{\alpha}(P)\right\|<\frac{\varepsilon}{2\left(3^{n}-1\right)}$. Finally, for each $\beta \neq o$, choose $\alpha(\beta)$ such that $\alpha(\beta) \lessdot o$ and $\beta \leq \alpha(\beta)$. Since $\xi_{\beta}(Q) \leq \xi(\alpha(\beta) \cdot Q) \leq 1$ and since $\mathcal{L}$ contains $3^{n}$ elements, one obtains

$$
\left\|B-\xi_{o}\left(\frac{Q}{r^{\prime}}\right) B-\sum_{\beta \neq o} \sum_{k=1}^{N_{\alpha(\beta)}} \xi_{\beta}\left(\frac{Q}{r^{\prime}}\right) f_{k}^{\alpha(\beta)}(Q) h_{k}^{\alpha(\beta)}(P)\right\|<\varepsilon .
$$

By semi-compactness of $B, \xi_{o}\left(\frac{Q}{r^{\prime}}\right) B$ belongs to $\mathcal{K}(\mathcal{H})$, and hence to $\mathfrak{C}$; and each term in the sum belongs to $\mathfrak{C}$ by construction. Since $\mathfrak{C}$ is norm closed, one gets that $B \in \mathfrak{C}$. 
d) For each $\alpha \lessdot o$, the uniqueness of $B_{\alpha}$ is shown by proving the following statement: if $C$ belongs to $\left\langle C\left(\overline{\mathbb{R}}^{\alpha}\right) \cdot C_{0}\left(\mathbb{R}^{n *}\right)\right\rangle$ and satisfies the condition $\lim _{r \rightarrow \infty}\left\|\xi\left(\alpha \cdot \frac{Q}{r}\right) C\right\|=0$, then $C=0$. To see this, assume that $C \neq 0$ and for simplicity let us fix $\alpha=(1,0, \ldots, 0)$. Choose $\varphi \in \mathcal{H}$ such that $\|\varphi\|=1$ and $C \varphi \neq 0$. By hypothesis, there exists $r \geq 1$ such that $\left\|\xi\left(\frac{Q_{1}}{r}\right) C\right\|<\frac{1}{2}\|C \varphi\|$, and so $\left\|\xi\left(\frac{Q_{1}}{r}\right) C e^{-i y P_{1}} \varphi\right\|<\frac{1}{2}\|C \varphi\|$ for each $y \in \mathbb{R}$. But, since all elements of $\left\langle C\left(\overline{\mathbb{R}}^{\alpha}\right) \cdot C_{0}\left(\mathbb{R}^{n *}\right)\right\rangle$ commute with the unitary operator $e^{-i y P_{1}}(y \in \mathbb{R})$, one has $\left\|\xi\left(\frac{Q_{1}}{r}\right) C e^{-i y P_{1}} \varphi\right\|=\left\|\xi\left(\frac{Q_{1}}{r}+\frac{y}{r}\right) C \varphi\right\| \rightarrow\|C \varphi\|$ as $y \rightarrow \infty$, a contradiction with the preceding inequality. Hence $C=0$.

Lemma 4.2. $\quad$ Let $H$ be a self-adjoint operator in $\mathcal{H}$ with domain $D(H)$ and assume that for each $\alpha \lessdot o$, there exists a self-adjoint operator $H_{\alpha}$ in $\mathcal{H}$, affiliated to $\left\langle C\left(\overline{\mathbb{R}}^{\alpha}\right) \cdot C_{0}\left(\mathbb{R}^{n *}\right)\right\rangle$, with domain equal to $D(H)$. Assume also that for some asymptotic localization function $\xi$ and each $\alpha \lessdot o$,

$$
\lim _{r \rightarrow \infty}\left\|\xi\left(\alpha \cdot \frac{Q}{r}\right)\left(H-H_{\alpha}\right)\right\|_{D(H) \rightarrow \mathcal{H}}=0 .
$$

Then $H$ is affiliated to $\mathfrak{C}$ and $\Pi_{\alpha}(H)=H_{\alpha}$ (in the sense of Definition 2.1).

Proof. Set $R:=(H-z)^{-1}$ and $R_{\alpha}:=\left(H_{\alpha}-z\right)^{-1}$ for any fixed $z \in \mathbb{C} \backslash \mathbb{R}$ and each $\alpha \lessdot o$. Since $H_{\alpha}$ is affiliated to the subalgebra $\left\langle C\left(\overline{\mathbb{R}}^{\alpha}\right) \cdot C_{0}\left(\mathbb{R}^{n *}\right)\right\rangle$ of $\mathfrak{C}, R_{\alpha}$ belongs to $\mathfrak{C}$ and thus is semi-compact, $c f$. i) of Lemma 4.1. Then $R$ is semi-compact since for any $\zeta \in C_{0}\left(\mathbb{R}^{n}\right), \zeta(Q) R_{\alpha} \in \mathcal{K}(\mathcal{H})$ and $R_{\alpha}^{-1} R$ is bounded by the closed graph theorem [11].

Furthermore,

$$
\left\|\xi\left(\alpha \cdot \frac{Q}{r}\right)\left(R-R_{\alpha}\right)\right\| \leq c\left\{\left\|\xi\left(\alpha \cdot \frac{Q}{r}\right)\left(H-H_{\alpha}\right) R\right\|+\left\|\left[\xi\left(\alpha \cdot \frac{Q}{r}\right), R_{\alpha}\right]\right\|\right\}
$$

where $c=\max \left\{\left\|R_{\alpha}\right\|,\left\|\left(H-H_{\alpha}\right) R\right\|\right\}$. The first term on the r.h.s. goes to 0 as $r \rightarrow \infty$ by hypothesis. For the second term, one has to use the isomorphism $\left\langle C\left(\overline{\mathbb{R}}^{\alpha}\right) \cdot C_{0}\left(\mathbb{R}^{n *}\right)\right\rangle \cong C_{0}\left(\mathbb{R}^{\alpha^{\perp} *}\right) \otimes \mathfrak{C}^{\alpha}$ introduced in Section 3 and either Lemma 3.4 of $[7]$ or a commutator expansion for terms of the form $\left[\xi\left(\alpha \cdot \frac{Q}{r}\right), g(\alpha \cdot P)\right]$ with $g \in C_{0}\left(\mathbb{R}^{\alpha^{\perp}}\right)$. It then follows that $\lim _{r \rightarrow \infty}\left\|\left[\xi\left(\alpha \cdot \frac{Q}{r}\right), R_{\alpha}\right]\right\|=0$, and the affiliation of $H$ to $\mathfrak{C}$ is obtained with Lemma 4.1 and the observation made before Definition 2.1.

We have thus obtained that $\Pi_{\alpha}\left((H-z)^{-1}\right)=\left(H_{\alpha}-z\right)^{-1}$ for all $z \in \mathbb{C} \backslash \mathbb{R}$. The last statement of the lemma follows from the density in $C_{0}(\mathbb{R})$ of the vector space generated by the set of functions $\left\{(\cdot-z)^{-1} \mid z \in \mathbb{C} \backslash \mathbb{R}\right\}$. 
We now give the general definition of the potentials under consideration, and we shall prove in Proposition 4.1 the affiliation to $\mathfrak{C}$ for the corresponding Schrödinger operators. One notices that if $V$ belongs to $C\left(\overline{\mathbb{R}}^{n}\right)$, the functions $V^{\alpha}$ introduced in the following definition are nothing but the restrictions of $V$ to the hypersurfaces $\overline{\mathbb{R}}^{\alpha}$.

Definition 4.1. A Borel function $V: \mathbb{R}^{n} \rightarrow \mathbb{R}$ is a cartesian potential (relative to $\mathcal{L}$ ) if there exists a collection of Borel functions $\left\{V^{\alpha}\right\}_{\alpha \in \mathcal{L}}$, with $V^{o} \equiv V$ and $V^{\alpha}: \mathbb{R}^{\alpha} \rightarrow \mathbb{R}$, such that for each $\alpha \in \mathcal{L}:$

i) $V^{\alpha}(Q)$ is $\boldsymbol{\Delta}^{\alpha}$-bounded with relative bound less than one,

ii) $\lim _{r \rightarrow \infty}\left\|\xi\left((\beta-\alpha) \cdot \frac{Q}{r}\right)\left(V^{\alpha}(Q)-V^{\beta}(Q)\right)\right\|_{\mathcal{H}_{\alpha}^{2} \rightarrow \mathcal{H}_{\alpha}}=0$ for each $\beta \lessdot \alpha$ and some asymptotic localization function $\xi$.

The second condition means that for each $\alpha \in \mathcal{L}$, the function $V^{\alpha}$ defined on $\mathbb{R}^{\alpha}$ approaches its asymptotic limits $V^{\beta}$ with $\beta \lessdot \alpha$ in the norm $\|\cdot\|_{\mathcal{H}_{\alpha}^{2} \rightarrow \mathcal{H}_{\alpha}}$. Let us observe that Lemma 9.4.8 of [1] implies that if $V$ is a cartesian potential, then $V^{\alpha}(Q)$ is $\boldsymbol{\Delta}$-bounded with relative bound less than one, and ii) is also fulfilled with the norm $\|\cdot\|_{\mathcal{H}^{2} \rightarrow \mathcal{H}}$ instead of the norm $\|\cdot\|_{\mathcal{H}_{\alpha}^{2} \rightarrow \mathcal{H}_{\alpha}}$. We give a rather general example of such potentials.

Example 1. Let $V_{1}$ be a bounded real function on $\mathbb{R}^{n}$ such that for each $\alpha \lessdot o$, there exists $V^{\alpha} \in L^{\infty}\left(\mathbb{R}^{\alpha}\right)$ satisfying

$$
\lim _{r \rightarrow \infty} \sup _{x \in \mathbb{R}^{n}}\left|\xi\left(\alpha \cdot \frac{x}{r}\right)\left(V_{1}(x)-V^{\alpha}(x)\right)\right|=0
$$

for some asymptotic localization function $\xi$. Let $V_{2}(Q)$ be a $\Delta$-bounded operator (relative bound less than one) such that $\lim _{r \rightarrow \infty}\left\|\xi\left(\frac{|Q|}{r}\right) V_{2}(Q)\right\|_{\mathcal{H}^{2} \rightarrow \mathcal{H}}=0$. Then $V^{o}:=V_{1}+V_{2}$ is a cartesian potential. Indeed, let $\beta \in \mathcal{L}$ with $|\beta|=n-2$ and let $\alpha, \alpha^{\prime}$ be the only two distinct elements of $\mathcal{L}$ such that $\beta \lessdot \alpha$ and $\beta \lessdot \alpha^{\prime}$. Let $j, j^{\prime} \in\{1, \ldots, n\}$ be such that $(\beta-\alpha)_{j} \neq 0$ and $\left(\beta-\alpha^{\prime}\right)_{j^{\prime}} \neq 0$. Then one can check that $\left\{\left.V^{\alpha}\right|_{x_{j}=\beta_{j} n}\right\}_{n \in \mathbb{N}}$ and $\left\{\left.V^{\alpha^{\prime}}\right|_{x_{j^{\prime}}=\beta_{j^{\prime}} n}\right\}_{n \in \mathbb{N}}$ are two Cauchy sequences in $L^{\infty}\left(\mathbb{R}^{\beta}\right)$ which converge to the same element (denoted $V^{\beta}$ ). Both requirements of Definition 4.1 are now clearly satisfied for $\alpha=o$ and for each $\alpha \lessdot o$. The same procedure can then be applied again in order to construct successively $V^{\beta}$ for all $\beta \in \mathcal{L}$ and to check that the conditions of Definition 4.1 are satisfied.

For the next proof and some later uses, we introduce the semilattice $\mathcal{L}^{\alpha}:=$ $\{\beta \in \mathcal{L} \mid \beta \leq \alpha\}$. 
Proposition 4.1. Assume that $V$ is a cartesian potential. Then $H=$ $-\boldsymbol{\Delta}+V$ is a cartesian Hamiltonian and $\Pi_{\alpha}(H)=-\boldsymbol{\Delta}+V^{\alpha}$ for each $\alpha \in \mathcal{L}$.

Proof. Since the proof is performed by induction over the lattice $\mathcal{L}$, let us first introduce some notations. For each $\alpha \in \mathcal{L}$ and each $\beta \leq \alpha$, let $\pi_{\beta}^{\alpha}: C\left(\overline{\mathbb{R}}^{\alpha}\right) \ni f \mapsto \pi_{\beta}^{\alpha}(f) \in C\left(\overline{\mathbb{R}}^{\beta}\right)$ be the covariant morphism given by restriction of $f$ to the hypersurface $\overline{\mathbb{R}}^{\beta}$, and let $\Pi_{\beta}^{\alpha}$ be the unique morphism $\mathfrak{C}^{\alpha} \rightarrow\left\langle C\left(\overline{\mathbb{R}}^{\beta}\right) \cdot C_{0}\left(\mathbb{R}^{\alpha *}\right)\right\rangle$ satisfying $\Pi_{\beta}^{\alpha}[f(Q) h(P)]=\pi_{\beta}^{\alpha}(f)(Q) h(P)$ for each $f \in C\left(\overline{\mathbb{R}}^{\alpha}\right)$ and each $h \in C_{0}\left(\mathbb{R}^{\alpha}\right)$. If $\alpha=o$, then $\Pi_{\beta}^{\alpha}$ is just $\Pi_{\beta}$. In this setting, the statement of the proposition reads: if $V^{\alpha}$ is a cartesian potential relative to the lattice $\mathcal{L}^{\alpha}$, then $H^{\alpha}=-\boldsymbol{\Delta}^{\alpha}+V^{\alpha}$ is affiliated to $\mathfrak{C}^{\alpha}$ and $\Pi_{\beta}^{\alpha}\left(H^{\alpha}\right)=-\Delta^{\alpha}+V^{\beta}$ for each $\beta \in \mathcal{L}^{\alpha}$.

Let us notice that for each $\alpha \in \mathcal{L}, V^{\alpha}$ is a cartesian potential relative to the lattice $\mathcal{L}^{\alpha}$. In the special case $|\alpha|=0, V^{\alpha}$ is a real number, $H^{\alpha}=V^{\alpha}$ and $H^{\alpha}$ is clearly affiliated to $\mathfrak{C}^{\alpha}$, which is simply $\mathbb{C}$. For any fixed $\alpha,|\alpha| \neq 0$, we may now assume that the assertions of the proposition are proved for each $H^{\beta}$ with $\beta \lessdot \alpha$ and we prove it for $H^{\alpha}$. With no loss of generality and for simplicity of notations, we choose $\alpha=o$.

So assume that for each $\beta \lessdot o, H^{\beta}$ is affiliated to $\mathfrak{C}^{\beta}$. Let $j \in\{1, \ldots, n\}$ such that $\beta_{j} \neq 0$. Then the operator $-\boldsymbol{\Delta}_{j} \otimes I+I \otimes H^{\beta}$ in $L^{2}\left(\mathbb{R}_{j}\right) \otimes \mathcal{H}_{\beta}$ is affiliated to the $\mathrm{C}^{*}$-algebra $C_{0}\left(\mathbb{R}_{j}^{*}\right) \otimes \mathfrak{C}^{\beta}$ (see [1], Section 8.2.3). Furthermore, if we identify $L^{2}\left(\mathbb{R}_{j}\right) \otimes \mathcal{H}_{\beta}$ with $\mathcal{H}$, then the operator $-\boldsymbol{\Delta}_{j} \otimes I+I \otimes H^{\beta}$ is equal to $-\boldsymbol{\Delta}+V^{\beta}$, which is thus affiliated to $\left\langle C\left(\overline{\mathbb{R}}^{\beta}\right) \cdot C_{0}\left(\mathbb{R}^{n *}\right)\right\rangle$, cf. Section 3. Hence $H_{\beta}=-\boldsymbol{\Delta}+V^{\beta}$ is a self-adjoint operator in $\mathcal{H}$ of domain $\mathcal{H}^{2}$ and affiliated to $\left\langle C\left(\overline{\mathbb{R}}^{\beta}\right) \cdot C_{0}\left(\mathbb{R}^{n *}\right)\right\rangle$. But $H$ is also a self-adjoint operator with domain $\mathcal{H}^{2}$ and $\left\|\xi\left(\beta \cdot \frac{Q}{r}\right)\left(H-H_{\beta}\right)\right\|_{\mathcal{H}^{2} \rightarrow \mathcal{H}}=\left\|\xi\left(\beta \cdot \frac{Q}{r}\right)\left(V(Q)-V^{\beta}(Q)\right)\right\|_{\mathcal{H}^{2} \rightarrow \mathcal{H}} \rightarrow 0$ as $r \rightarrow \infty$ ( $\xi$ is the asymptotic localization function introduced in Definition 4.1). By invoking Lemma 4.2 one gets that $H$ is affiliated to $\mathfrak{C}$ and that $\Pi_{\beta}(H)=H_{\beta}$ for each $\beta \lessdot o$.

The general case $\Pi_{\gamma}(H)=H_{\gamma}$ for all $\gamma \leq o$ is obtained by taking into account the trivial equality $\Pi_{\gamma}=\Pi_{\gamma} \circ \Pi_{\beta}$ for $\gamma \leq \beta$, the identification of the morphisms $\Pi_{\gamma}$ on $\left\langle C\left(\overline{\mathbb{R}}^{\beta}\right) \cdot C_{0}\left(\mathbb{R}^{n *}\right)\right\rangle$ and $I \otimes \Pi_{\gamma}^{\beta}$ on $C_{0}\left(\mathbb{R}^{\beta^{\perp} *}\right) \otimes \mathfrak{C}^{\beta}$, and by using the assumption $\Pi_{\gamma}^{\beta}\left(H^{\beta}\right)=-\boldsymbol{\Delta}^{\beta}+V^{\gamma}$.

\section{§5. The Mourre Estimate and the Limiting Absorption Principle}

The strategy for obtaining the results announced in this title is similar to that developed for the N-body problem. The analogy is possible mainly 
because of the rich internal structure of $\mathfrak{C}$ and its compatibility with the unitary group $\left\{W_{t}\right\}_{t \in \mathbb{R}}$ of dilations in $\mathcal{H}$ (Lemma 5.1). More precisely, we consider $\left\{W_{t}=e^{2 i A t}\right\}_{t \in \mathbb{R}}$ with self-adjoint generator $A=\frac{1}{4} \sum_{j=1}^{n}\left(P_{j} Q_{j}+Q_{j} P_{j}\right)$. Let us observe that $[\boldsymbol{\Delta}, i A]=\boldsymbol{\Delta}$, and recall that this group leaves $\mathcal{H}^{2}$ invariant. Each $W_{t}$ induces an automorphism $\mathcal{W}_{t}$ of $\mathcal{B}(\mathcal{H})$, namely $\mathcal{W}_{t}[B]=W_{-t} B W_{t}$ for $B \in \mathcal{B}(\mathcal{H})$.

Lemma 5.1. For each $t \in \mathbb{R}, \mathcal{W}_{t}$ leaves the $C^{*}$-algebra $\mathfrak{C}$ invariant; for each $\alpha \in \mathcal{L}, \mathcal{W}_{t} \circ \Pi_{\alpha}=\Pi_{\alpha} \circ \mathcal{W}_{t}$ on $\mathfrak{C}$.

Proof. For any $f \in C\left(\overline{\mathbb{R}}^{n}\right)$ and any $h \in C_{0}\left(\mathbb{R}^{n}\right)$, one has $\mathcal{W}_{t}[f(Q) h(P)]=$ $f\left(e^{-t} Q\right) h\left(e^{t} P\right)$. It is now easy to verify that $\left(\mathcal{W}_{t} \circ \Pi_{\alpha}\right)[f(Q) h(P)]=\left(\Pi_{\alpha} \circ\right.$ $\left.\mathcal{W}_{t}\right)[f(Q) h(P)]$. Since $\mathfrak{C}$ is the norm closure of the vector space generated by such products, $\mathfrak{C}$ is invariant and $\mathcal{W}_{t}$ and $\Pi_{\alpha}$ commute on $\mathfrak{C}$.

The next lemma contains two results which are analogous to the statements of Lemma 9.4.3 and Theorem 8.4.3 of [1]. The compatibility of the structure of $\mathfrak{C}$ with the dilation group is essential. One observes that $A=$ $\frac{1}{4} \sum_{\left\{j \mid \alpha_{j}=0\right\}}\left(P_{j} Q_{j}+Q_{j} P_{j}\right)+\frac{1}{4} \sum_{\left\{j \mid \alpha_{j} \neq 0\right\}}\left(P_{j} Q_{j}+Q_{j} P_{j}\right) \equiv A^{\alpha}+A^{\alpha^{\perp}}$, where $A^{\alpha}$ (resp. $\left.A^{\alpha^{\perp}}\right)$ is the generator of dilations in $\mathbb{R}^{\alpha}$ (resp. $\mathbb{R}^{\alpha^{\perp}}$ ).

Lemma 5.2. Let $V$ be a cartesian potential such that $H=-\boldsymbol{\Delta}+V$ is of class $C_{u}^{1}(A)$. Then:

i) for each $\alpha \in \mathcal{L}, H_{\alpha}$ is of class $C_{u}^{1}(A)$ and $H^{\alpha}$ is of class $C_{u}^{1}\left(A^{\alpha}\right)$,

ii) one has $\tilde{\varrho}_{H}^{A}=\min _{\alpha \lessdot o} \varrho_{H_{\alpha}}^{A}$.

The proof of i) can be performed by rewriting the proof of Lemma 9.4.3 of [1] in our formalism. The statement ii) is obtained by taking into account the injective morphism (3), together with our Lemma 5.1 and Proposition 8.3.5 of $[1]$.

Let us now recall two important results in Mourre theory which are expressed in terms of the functions $\varrho$ and $\tilde{\varrho}$. Proofs can be found in Section 7.2 of $[1]$.

Proposition 5.1. Let $H$ be a self-adjoint operator of class $C^{1}(A)$.

i) If $\tilde{\varrho}_{H}^{A}(\lambda)>0$ for some $\lambda \in \mathbb{R}$, then $\lambda$ has a neighbourhood in which there is at most a finite number of eigenvalues of $H$, each of finite multiplicity.

ii) One has $\varrho_{H}^{A}(\lambda)=\tilde{\varrho}_{H}^{A}(\lambda)$ unless $\lambda$ is an eigenvalue of $H$ and $\tilde{\varrho}_{H}^{A}(\lambda)>0$, in which case $\varrho_{H}^{A}(\lambda)=0$. 
The next statement contains the Mourre estimate, which is the main result of this section.

Proposition 5.2. Assume that $V$ is a cartesian potential and that $H=$ $-\boldsymbol{\Delta}+V$ is of class $C_{u}^{1}(A)$. Then $\tau(H)$ and $\kappa(H)$ are closed countable sets, the eigenvalues of $H$ outside $\tau(H)$ are of finite multiplicity and can accumulate only at points belonging to $\tau(H)$, and $\mu^{A}(H)$ is equal to $\mathbb{R} \backslash \kappa(H)$. Moreover, for each $\lambda \in \mathbb{R}$,

$$
\tilde{\varrho}_{H}^{A}(\lambda)=\inf \{\lambda-\mu \mid \mu \in \tau(H), \mu \leq \lambda\}
$$

with the convention that the infimum over an empty set is $+\infty$.

Proof. a) We begin with some preliminary observations. One notices that for each $\alpha \in \mathcal{L}, \tau\left(H^{\alpha}\right)=\cup_{\beta<\alpha} \sigma_{p}\left(H^{\beta}\right)$ and $\kappa\left(H^{\alpha}\right)=\cup_{\beta \leq \alpha} \sigma_{p}\left(H^{\beta}\right)$ are countable sets. Furthermore, if $\tilde{\varrho}_{H^{\alpha}}^{A^{\alpha}}(\lambda)>0$ for all $\lambda \in \mathbb{R} \backslash \tau\left(H^{\alpha}\right)$, then $\sigma_{p}\left(H^{\alpha}\right)$ can only accumulate at points of $\tau\left(H^{\alpha}\right)$, since otherwise it would contradict i) of Proposition 5.1. Since $\kappa\left(H^{\alpha}\right)$ is equal to $\tau\left(H^{\alpha}\right) \cup \sigma_{p}\left(H^{\alpha}\right)$, this implies that if $\tau\left(H^{\alpha}\right)$ is closed and $\tilde{\varrho}_{H^{\alpha}}^{A^{\alpha}}(\lambda)>0$ for all $\lambda \in \mathbb{R} \backslash \tau\left(H^{\alpha}\right)$, then $\kappa\left(H^{\alpha}\right)$ is also closed.

b) The proof is going to be performed by induction over $\mathcal{L}$. We have already noticed that for each $\alpha \in \mathcal{L}, V^{\alpha}$ is a cartesian potential relative to $\mathcal{L}^{\alpha}$ and $H^{\alpha}$ is of class $C_{u}^{1}\left(A^{\alpha}\right)$. First, for $|\alpha|=0, H^{\alpha}$ is equal to the real number $V^{\alpha}, \tau\left(H^{\alpha}\right)=\emptyset$ and $\kappa\left(H^{\alpha}\right)=\left\{V^{\alpha}\right\}$. Moreover, $\varrho_{H^{\alpha}}^{0}(\lambda)=+\infty$ for all $\lambda \in \mathbb{R}$, while $\varrho_{H^{\alpha}}^{0}\left(V^{\alpha}\right)=0$ and $\varrho_{H^{\alpha}}^{0}(\lambda)=+\infty$ for $\lambda \neq V^{\alpha}$. The proposition is thus verified in this special case. Next, for any fixed $\alpha,|\alpha| \neq 0$, we assume that the assertions of the proposition are proved for each $H^{\beta}$ with $\beta \lessdot \alpha$ and we prove it for $H^{\alpha}$. For simplicity but with no loss of generality, we choose $\alpha=o$.

c) We start by determining $\varrho_{H_{\beta}}^{A}$ for each $\beta \lessdot o$. If $j \in\{1, \ldots, n\}$ such that $\beta_{j} \neq 0$, then $H_{\beta}$ is equal to $-\boldsymbol{\Delta}_{j} \otimes I+I \otimes H^{\beta}$. The $\varrho$-function for operators of this form is extensively studied in [1], Theorem 8.3.6:

$$
\varrho_{H_{\beta}}^{A}(\lambda)=\inf _{\lambda_{1}+\lambda_{2}=\lambda}\left\{\varrho_{H^{\beta}}^{A^{\beta}}\left(\lambda_{1}\right)+\varrho_{-\Delta_{j}}^{A^{\beta^{\perp}}}\left(\lambda_{2}\right)\right\} .
$$

But $\varrho_{-\Delta_{j}}^{A^{\beta^{\perp}}}\left(\lambda_{2}\right)=\infty$ if $\lambda_{2}<0$ and $\varrho_{-\Delta_{j}}^{A^{\beta^{\perp}}}\left(\lambda_{2}\right)=\lambda_{2}$ if $\lambda_{2} \geq 0$, and hence $\varrho_{H_{\beta}}^{A}(\lambda)=\inf _{\mu \leq \lambda}\left\{\varrho_{H^{\beta}}^{A^{\beta}}(\mu)+(\lambda-\mu)\right\}$. By assumption, $\tilde{\varrho}_{H^{\beta}}^{A^{\beta}}(\lambda)=\inf \{\lambda-$ $\left.\mu \mid \mu \in \tau\left(H^{\beta}\right), \mu \leq \lambda\right\}$, and so $\tilde{\varrho}_{H^{\beta}}^{A^{\beta}}$ is zero on $\tau\left(H^{\beta}\right)$ and strictly positive on $\mathbb{R} \backslash \tau\left(H^{\beta}\right)$ (since $\tau\left(H^{\beta}\right)$ is assumed to be closed). Thus, in view of ii) of Proposition 5.1, $\varrho_{H^{\beta}}^{A^{\beta}}(\lambda)=0$ if $\lambda \in \kappa\left(H^{\beta}\right)$ and $\varrho_{H^{\beta}}^{A^{\beta}}(\lambda)=\varrho_{H^{\beta}}^{A^{\beta}}(\lambda)$ elsewhere. From 
these relations one easily finds that $\varrho_{H_{\beta}}^{A}(\lambda)=\inf \left\{\lambda-\mu \mid \mu \in \kappa\left(H^{\beta}\right), \mu \leq \lambda\right\}$ for each $\beta \lessdot o$.

d) By using the result of c) and ii) of Lemma 5.2, one gets that $\tilde{\varrho}_{H}^{A}(\lambda)=$ $\inf \{\lambda-\mu \mid \mu \in \tau(H), \mu \leq \lambda\}$, because $\cup_{\beta \lessdot o} \kappa\left(H^{\beta}\right)=\cup_{\beta \lessdot o}\left[\cup_{\gamma \leq \beta} \sigma_{p}\left(H^{\gamma}\right)\right]=$ $\tau(H)$. Since $\tau(H)$ is a finite union of closed sets, it is closed. Hence $\tilde{\varrho}_{H}^{A}$ is strictly positive outside $\tau(H)$ and zero on $\tau(H)$, so that one may apply the result of a) with $\alpha=o$. By taking into account the statement ii) of Proposition 5.1 , one sees that $\varrho_{H}^{A}(\lambda)=0$ if $\lambda \in \kappa(H)$ and $\varrho_{H}^{A}(\lambda)>0$ if $\lambda \notin \kappa(H)$. Hence $\mu^{A}(H)=\mathbb{R} \backslash \kappa(H)$.

Collecting the results obtained so far, we can now prove Theorem 1.1. We mention that if the operator $H$ is of class $C^{1,1}(A)$, the $C_{u}^{1}(A)$-condition of Proposition 5.2 is fulfilled.

Proof of Theorem 1.1. Since the potential $V$ is $\boldsymbol{\Delta}$-bounded (with relative bound less than one) and is of class $C^{1,1}(A), H$ is of class $C^{1,1}(A)$. From (4), one has $\sigma_{\text {ess }}(H)=\cup_{\alpha \lessdot o} \sigma\left(H_{\alpha}\right)$. But $\sigma\left(H_{\alpha}\right)=\left[\inf \sigma\left(H^{\alpha}\right), \infty\right)$ because $H_{\alpha}=-\boldsymbol{\Delta}_{j} \otimes I+I \otimes H^{\alpha}$ for $j$ such that $\alpha_{j} \neq 0$, cf. [17]. This implies i). ii) is part of Proposition 5.2. iv) results from our Proposition 5.2 and Proposition 7.4.6 of [1]. Finally, iii) is a well-known consequence of iv).

In order to ascertain that the $C^{1,1}(A)$-condition is not too restrictive with respect to the cartesian anisotropy, let us indicate two examples of cartesian potentials of class $C^{1,1}(A)$.

Example 2. For any cartesian potential $\widetilde{V}$, we consider the following approximation $V_{m}$ of $\widetilde{V}$. Let $m$ be any positive number and $\xi$ an asymptotic localization function. Set $\xi_{0}(y):=1-\xi(y)-\xi(-y)$ for $y \in \mathbb{R}$ and $\xi_{\alpha}(x):=$ $\prod_{\left\{j \mid \alpha_{j} \neq 0\right\}} \xi\left(\alpha_{j} x_{j}\right) \prod_{\left\{k \mid \alpha_{k}=0\right\}} \xi_{0}\left(x_{k}\right)$ for $x \in \mathbb{R}^{n}$ and $\alpha \in \mathcal{L}$. We now define $V_{m}(x):=\sum_{\alpha \in \mathcal{L}} \xi_{\alpha}\left(\frac{x}{m}\right) \widetilde{V}^{\alpha}(x)$ for $x \in \mathbb{R}^{n}$. Some calculations show that $V_{m}$ is a Mourre potential (cf. Definition 2.2) and that $\lim _{m \rightarrow \infty}\left\|\widetilde{V}(Q)-V_{m}(Q)\right\|_{\mathcal{H}^{2} \rightarrow \mathcal{H}}=$ 0 . Let $V$ be equal to $V_{m}+V_{\mathrm{LR}}+V_{\mathrm{SR}}$, with $V_{\mathrm{LR}}\left(V_{\mathrm{SR}}\right)$ a long-range (short-range) potential satisfying $\lim _{r \rightarrow \infty}\left\|\xi\left(\frac{|Q|}{r}\right)\left(V_{\mathrm{LR}}(Q)+V_{\mathrm{SR}}(Q)\right)\right\|_{\mathcal{H}^{2} \rightarrow \mathcal{H}}=0$. Then $V$ is a cartesian potential of class $C^{1,1}(A)$.

Example 3. Let $V_{j}$ be a bounded function on $\mathbb{R}_{j}$ having limits as $x_{j} \rightarrow$ $\pm \infty$ and converging to these limits in a short-range or long-range way. Then $V:=\prod_{j=1}^{n} V_{j}$ is a cartesian potential of class $C^{1,1}(A)$. To check this assertion, we first observe that if $U$ is a self-adjoint operator in $\mathcal{H}_{\alpha}$ of class $C^{1,1}\left(A^{\alpha}\right)$ for some $\alpha \in \mathcal{L}$, then $U$ is also of class $C^{1,1}(A)$. Furthermore, the product of a finite 
number of bounded potentials of class $C^{1,1}(A)$ belongs to the same class (this is easily proved by using Lemma 6.2.1 and Proposition 5.2.3 of [1]). Finally, both requirement of definition 4.1 are satisfied by the potential $V$, which is therefore cartesian. Note that the example given in the Introduction is of this type.

\section{$\S 6 . \quad$ The Asymptotic Velocity}

In this section, we prove the existence of the asymptotic velocity and state some of its properties. This velocity is going to play an essential role in the definition of the wave operators. Most of the results of this section are inspired or adapted from Section 6.6 of [4]. However, since cartesian potentials and Nbody potentials differ substantially, none of the result of this reference can be directly quoted. We refer to that book for further comments on the asymptotic velocity and other applications.

Proposition 6.1. Let $V$ be a cartesian potential such that $H=-\boldsymbol{\Delta}+V$ is of class $C_{u}^{1}(A)$. Assume also that for each $j \in\{1, \ldots, n\}$ and for some asymptotic localization function $\xi$,

$$
\int_{0}^{\infty}\left\|\xi\left( \pm \frac{Q_{j}}{r}\right) \nabla_{j} V(Q)\right\|_{\mathcal{H}^{2} \rightarrow \mathcal{H}^{-2}} d r<\infty .
$$

Then,

i) there exists a n-tuple $\mathcal{P}$ of commuting self-adjoint operators such that for all $f \in C_{0}\left(\mathbb{R}^{n}\right)$ :

$$
s-\lim _{t \rightarrow+\infty} e^{i H t} f\left(\frac{Q}{2 t}\right) e^{-i H t}=f(\mathcal{P})
$$

ii) for each $\eta \in C_{0}(\mathbb{R})$ and each $f \in C_{0}\left(\mathbb{R}^{n}\right),[\eta(H), f(\mathcal{P})]=0$, i.e. the asymptotic velocity $\mathcal{P}$ commutes with the Hamiltonian $H$,

iii) the subspace of the states with zero asymptotic velocity is equal to the subspace spanned by the eigenvectors of $H$.

Since the limit $t \rightarrow-\infty$ could be handled similarly, we simply do not consider it. The entire section is devoted to the proof of this proposition and therefore, unless otherwise stated, it is always assumed that the potential $V$ satisfies its hypotheses. The proofs involve a considerable number of commutator computations, which will of course not be presented in full details. We 
start with some considerations on the notations and with a technical lemma that will be used freely subsequently.

Let us consider an operator-valued mapping $\Phi:[1, \infty) \ni t \mapsto \Phi(t) \in$ $\mathcal{B}(\mathcal{H})$. If there exists some constant $c<\infty$ such that $\|\Phi(t)\| \leq c$ for all $t \geq 1$, then $\Phi$ is said to be a bounded operator-valued mapping. We write $\Phi \in B C^{1}([1, \infty), \mathcal{B}(\mathcal{H}))$ if the mapping is bounded and differentiable in norm with bounded derivative. Let $m$ be any positive integer and $t \in[1, \infty)$. Then $\Phi$ (or by a slight abuse of notation $\Phi(t))$ belongs to $L^{m}((1, \infty), d t)$ if $\|\Phi(\cdot)\| \in$ $L^{m}((1, \infty), d t)$, to $o\left(t^{-m}\right)$ if $\lim _{t \rightarrow \infty} t^{m}\|\Phi(t)\|=0$, or to $O\left(t^{-m}\right)$ if $t^{m}\|\Phi(t)\| \leq$ $c<\infty$ for all $t \geq 1$. We say that $\Phi$ is integrable along the evolution (with respect to $H)$ if there exists a constant $c<\infty$ such that $\int_{1}^{\infty}\left|\left\langle e^{-i H t} \varphi, \Phi(t) e^{-i H t} \varphi\right\rangle\right| d t \leq$ $c\|\varphi\|^{2}$ for all $\varphi \in \mathcal{H}$.

For each $\alpha \in \mathcal{L}$ we define the open subset of $\mathbb{R}^{n}$ :

$$
Y_{\alpha}:=\left\{x \in \mathbb{R}^{n} \mid \alpha_{j} x_{j}>0 \text { for all } j \text { with } \alpha_{j} \neq 0\right\} .
$$

If $\alpha \lessdot o$ we also use the more familiar notation $Y_{j}^{ \pm}:=\left\{x \in \mathbb{R}^{n} \mid \pm x_{j}>0\right\}$ with $j$ and the sign \pm given by the only $\alpha_{j} \neq 0$. Let us make an obvious but very useful observation. For each $\alpha \in \mathcal{L}$ and each $f \in C_{c}\left(\mathbb{R}^{n}\right)$ with support in $Y_{\alpha}$ there exists $\delta>0$ such that $\prod_{\left\{j \mid \alpha_{j} \neq 0\right\}} \xi\left(\alpha_{j} \frac{x_{j}}{\delta}\right) f(x)=f(x)$ for any asymptotic localization function $\xi$ and all $x \in \mathbb{R}^{n}$.

In the sequel, unless explicitly mentioned, all functions $\eta, f, \ldots$ are assumed to be real.

Lemma 6.1. For each $\eta \in C_{c}^{\infty}(\mathbb{R})$, each $j \in\{1, \ldots, n\}$, each $\alpha \in \mathcal{L}$ and all $t \geq 1$, the following statements are true:

i) if $f$ is a twice differentiable function on $\mathbb{R}^{n}$ with bounded derivatives of order 0,1 and 2 , then $\left[\eta(H), f\left(\frac{Q}{2 t}\right)\right](H+i) \in O\left(t^{-1}\right)$,

ii) if $f \in C_{c}^{2}\left(\mathbb{R}^{n}\right)$ with support in $Y_{j}^{ \pm}$, then $\eta(H) f\left(\frac{Q}{2 t}\right) \nabla_{j} V(Q) \eta(H) \in$ $L^{1}((1, \infty), d t)$,

iii) if $f \in C_{c}\left(\mathbb{R}^{n}\right)$ with support in $Y_{j}^{ \pm}$, then $\left[P_{j}, \eta(H)\right] f\left(\frac{Q}{2 t}\right) \in L^{1}((1, \infty), d t) \cap$ $o\left(t^{0}\right)$,

iv) if $f \in C_{c}\left(\mathbb{R}^{n}\right)$ with support in $Y_{\alpha}$, then $f\left(\frac{Q}{2 t}\right)\left(\eta(H)-\eta\left(H_{\alpha}\right)\right) \in o\left(t^{0}\right)$,

v) the operator $\left(P_{j}+i\right) Q_{j} \eta(H)\left(1+Q^{2}\right)^{-1 / 2}$ belongs to $\mathcal{B}(\mathcal{H})$. 
The proof of this lemma is given in the Appendix. Let us however mention that the statement i) requires only the hypothesis that $V$ be $\Delta$-bounded with relative bound less than one. In line with iv), one could also prove some anisotropic non-propagation estimates at suitable energies. More general results of this type can be found in [2].

For each operator-valued mapping $\Phi \in B C^{1}([1, \infty), \mathcal{B}(\mathcal{H}))$ we define its Heisenberg derivative $\mathbf{D} \Phi$ : let $\varphi, \psi$ in $\mathcal{H}^{2}$ (the domain of $H$ ) and $t \geq 1$, then

$$
\langle\psi, \mathbf{D} \Phi(t) \varphi\rangle:=i\langle H \psi, \Phi(t) \varphi\rangle-i\langle\psi, \Phi(t) H \varphi\rangle+\left\langle\psi, \frac{d}{d t} \Phi(t) \varphi\right\rangle .
$$

One notices that $\left\langle\psi, e^{i H t} \mathbf{D} \Phi(t) e^{-i H t} \varphi\right\rangle$ is equal to $\frac{d}{d t}\left\langle\psi, e^{i H t} \Phi(t) e^{-i H t} \varphi\right\rangle$ for each $\varphi, \psi \in \mathcal{H}^{2}$. If, for each $t \geq 1, \mathbf{D} \Phi(t)$ extends continuously to a bounded operator (we keep the same notation for this extension), and if there exists some constant $c<\infty$ such that $\|\mathbf{D} \Phi(t)\| \leq c$ for all $t \geq 1$, then we write $\Phi \in B C_{H}^{1}([1, \infty), \mathcal{B}(\mathcal{H}))$. In our applications $\Phi(t)$ will often be equal $\eta(H) f\left(\frac{Q}{2 t}\right) \eta(H)$ with $\eta \in C_{c}^{\infty}(\mathbb{R})$ and $f$ a bounded function defined on $\mathbb{R}^{n}$. Then a sufficient condition such that $\Phi \in B C_{H}^{1}([1, \infty), \mathcal{B}(\mathcal{H}))$ is that $f \in C^{2}\left(\mathbb{R}^{n}\right)$ and that all first order partial derivatives of $f$ have a bounded support.

As mentioned in the Introduction, we shall prove various propagation estimates. For this purpose, we review two standard results that will be constantly used (proofs can be found in the Appendix).

Lemma 6.2. Consider a self-adjoint operator-valued mapping $\Phi$ belonging to $B C^{1}([1, \infty), \mathcal{B}(\mathcal{H}))$. Assume that there exist some bounded operatorvalued mappings $B, L, F$, with $L \in L^{1}((1, \infty), d t)$ and $F$ integrable along the evolution, such that one of the following two inequalities is satisfied for all $t \geq 1$ and each $\varphi \in \mathcal{H}^{2}$ :

$$
\pm\langle\varphi, \mathbf{D} \Phi(t) \varphi\rangle \geq\left\langle\varphi, B^{*}(t) B(t) \varphi\right\rangle+\langle\varphi, F(t) \varphi\rangle+\langle\varphi, L(t) \varphi\rangle
$$

Then there exists $c<\infty$ such that $\int_{1}^{\infty}\left\|B(t) e^{-i H t} \varphi\right\|^{2} d t \leq c\|\varphi\|^{2}$ for all $\varphi \in \mathcal{H}^{2}$.

Lemma 6.3. $\quad$ Consider an operator-valued mapping $\Phi$ which belongs to $B C^{1}([1, \infty), \mathcal{B}(\mathcal{H}))$, and let $H_{1}$ and $H_{2}$ be two self-adjoint operators in $\mathcal{H}$ of domain $\mathcal{D}_{1}$ and $\mathcal{D}_{2}$ respectively. Assume that there exist a finite integer $N$ and some bounded operator-valued mappings $E_{k}, F_{k}$ and $L$ such that for all $\varphi \in \mathcal{D}_{1}$, 
$\psi \in \mathcal{D}_{2}$ and all $t \geq 1$ :

$$
\begin{gathered}
\left|i\left\langle H_{2} \psi, \Phi(t) \varphi\right\rangle-i\left\langle\psi, \Phi(t) H_{1} \varphi\right\rangle+\left\langle\psi, \frac{d}{d t} \Phi(t) \varphi\right\rangle\right| \\
\leq \sum_{k=1}^{N}\left\|E_{k}(t) \psi\right\|\left\|F_{k}(t) \varphi\right\|+\|L(t)\|\|\psi\|\|\varphi\| .
\end{gathered}
$$

Assume furthermore that $L$ belongs to $L^{1}((1, \infty), d t)$ and that there is a constant $c<\infty$ such that for each $k \in\{1, \ldots, N\}, \int_{1}^{\infty}\left\|E_{k}(\tau) e^{-i H_{2} \tau} \psi\right\|^{2} d \tau \leq$ $c^{2}\|\psi\|^{2}$ for all $\psi \in \mathcal{D}_{2}$ and $\int_{1}^{\infty}\left\|F_{k}(\tau) e^{-i H_{1} \tau} \varphi\right\|^{2} d \tau \leq c^{2}\|\varphi\|^{2}$ for all $\varphi \in \mathcal{D}_{1}$. Then $s-\lim _{t \rightarrow \infty} e^{i H_{2} t} \Phi(t) e^{-i H_{1} t}$ exists.

In most of our applications $H_{1}$ and $H_{2}$ are equal to $H$, and therefore (7) is nothing but $|\langle\psi, \mathbf{D} \Phi(t) \varphi\rangle|$.

The next lemma contains two statements usually called maximal velocity estimates. Both are proved under the single assumption that the potential $V$ be $\Delta$-bounded with relative bound less than one. It slightly extends the validity of similar results obtained in [4].

To shorten some equations below and when the context leaves no doubt, the arguments of certain functions are not repeated all along the proofs.

Lemma 6.4. For each $\eta \in C_{c}^{\infty}(\mathbb{R})$, there exists a constant $c_{\eta}>0$ with the property that

i) for each $f \in C_{c}^{\infty}(\mathbb{R})$ with support in $\left(c_{\eta}, \infty\right)$, there exists $c<\infty$ such that for all $\varphi \in \mathcal{H}$,

$$
\int_{1}^{\infty}\left\|f\left(\frac{|Q|}{2 t}\right) \eta(H) e^{-i H t} \varphi\right\|^{2} \frac{d t}{t} \leq c\|\varphi\|^{2},
$$

ii) if $f$ is a $C^{\infty}$-function on $\mathbb{R}$ with support in $\left(c_{\eta}, \infty\right)$ and such that $f=1$ in a neighbourhood of $\infty$, then

$$
s-\lim _{t \rightarrow \infty} e^{i H t} \eta(H) f\left(\frac{|Q|}{2 t}\right) \eta(H) e^{-i H t}=0 .
$$

Proof. We fix a number $c_{\eta}^{\prime} \in \mathbb{R}$ such that $\operatorname{supp} \eta \subset\left(-\infty, c_{\eta}^{\prime}\right)$ and a function $\tilde{\eta} \in C_{c}^{\infty}\left(\left(-\infty, c_{\eta}^{\prime}\right)\right)$ satisfying $\tilde{\eta} \eta=\eta$ and $0 \leq \tilde{\eta} \leq 1$. We let $c_{\eta}^{\prime \prime}$ be a positive constant such that $\sup _{j}\left\|\chi_{\left(-\infty, c_{\eta}^{\prime}\right]}(H) P_{j}\right\| \leq c_{\eta}^{\prime \prime} / n$, where $\chi_{I}$ is the characteristic function of the interval $I$. We now fix the constant $c_{\eta}$ such that $c_{\eta}>c_{\eta}^{\prime \prime}$. 
a) For the proof of i), choose $\tilde{f} \in C_{c}^{\infty}(\mathbb{R})$ with support in $\left(c_{\eta}, \infty\right)$ and such that $\tilde{f} f=f$ and $0 \leq \tilde{f} \leq 1$. For $t \geq 1$, set $\Phi(t):=\eta(H) F\left(\frac{|Q|}{2 t}\right) \eta(H)$ with $F(s)=\int_{-\infty}^{s} f^{2}(\tau) d \tau$. Then $\Phi \in B C_{H}^{1}([1, \infty), \mathcal{B}(\mathcal{H}))$ and one has

$$
-\mathbf{D} \Phi(t)=\frac{1}{t} \eta\left\{\frac{|Q|}{2 t} f^{2}\left(\frac{|Q|}{2 t}\right)-\frac{1}{2} P \cdot \frac{Q}{|Q|} f^{2}\left(\frac{|Q|}{2 t}\right)-\frac{1}{2} f^{2}\left(\frac{|Q|}{2 t}\right) \frac{Q}{|Q|} \cdot P\right\} \eta .
$$

By using i) of Lemma 6.1, one can check that

$$
\tilde{\eta}(H) P \cdot \frac{Q}{|Q|} f^{2}+f^{2} \frac{Q}{|Q|} \cdot P \tilde{\eta}(H)=f\left\{\tilde{\eta} P \cdot \frac{Q}{|Q|} \tilde{f}+\tilde{f} \frac{Q}{|Q|} \cdot P \tilde{\eta}\right\} f+O\left(t^{-1}\right) .
$$

Our choice of $c_{\eta}^{\prime \prime}$ implies that $-c_{\eta}^{\prime \prime} \leq \frac{1}{2}\left\{\tilde{\eta} P \cdot \frac{Q}{|Q|} \tilde{f}+\tilde{f} \frac{Q}{|Q|} \cdot P \tilde{\eta}\right\} \leq c_{\eta}^{\prime \prime}$. Furthermore one has $\frac{|Q|}{2 t} f^{2}\left(\frac{|Q|}{2 t}\right) \geq c_{\eta} f^{2}\left(\frac{|Q|}{2 t}\right)$, and hence $-\mathbf{D} \Phi(t) \geq \frac{1}{t} \eta f\left\{c_{\eta}-c_{\eta}^{\prime \prime}\right\} f \eta+$ $O\left(t^{-2}\right)$. Since $c_{\eta}-c_{\eta}^{\prime \prime}>0$ and by observing that for any $\varphi \in \mathcal{H}, \tilde{\eta}(H) \varphi$ belongs to $\mathcal{H}^{2}$, the statement i) is seen to be a consequence of Lemma 6.2.

b) For $f$ as in i) or ii), let $\tilde{f} \in C_{c}^{\infty}(\mathbb{R})$ with support in $\left(c_{\eta}, \infty\right)$ be such that $\tilde{f} f^{\prime}=f^{\prime}$ and $0 \leq \tilde{f} \leq 1$. For $r \geq 1$ set $\Phi_{r}(t):=\eta(H) f\left(\frac{|Q|}{2 r t}\right) \eta(H)$ and observe that $\Phi_{r}$ is an operator-valued mapping belonging to $B C_{H}^{1}([1, \infty), \mathcal{B}(\mathcal{H}))$. As in a) above, one finds that

$$
\mathbf{D} \Phi_{r}(t)=\frac{1}{t} \eta \tilde{f}\left\{\frac{1}{2 r} \tilde{\eta} P \cdot \frac{Q}{|Q|} f^{\prime}+\frac{1}{2 r} f^{\prime} \frac{Q}{|Q|} \cdot P \tilde{\eta}-\frac{|Q|}{2 r t} f^{\prime}\right\} \tilde{f} \eta+O\left(t^{-2}\right) .
$$

All terms between brackets are norm bounded independently of $t$ for $t \geq$ 1. So by applying Lemma 6.3 and by using i) one gets the existence of $\mathrm{s}-\lim _{t \rightarrow \infty} e^{i H t} \Phi_{r}(t) e^{-i H t}$.

c) Let us show that this limit is zero if $f$ satisfies the hypothesis of i). Indeed, one may then choose $\tilde{f} \in C_{c}^{\infty}(\mathbb{R})$ with support in $\left(c_{\eta}, \infty\right)$ and such that $\tilde{f} f=f$. Then by i) there exists $c<\infty$ such that for all $\varphi \in \mathcal{H}$,

$$
\begin{aligned}
\int_{1}^{\infty}\left|\left\langle\varphi, e^{i H t} \Phi_{r}(t) e^{-i H t} \varphi\right\rangle\right| \frac{d t}{t} & \leq\|f\|_{L^{\infty}} \int_{1}^{\infty}\left\|\tilde{f}\left(\frac{|Q|}{2 r t}\right) \eta(H) e^{-i H t} \varphi\right\|^{2} \frac{d t}{t} \\
& \leq c\|\varphi\|^{2}
\end{aligned}
$$

This implies that $\lim _{t \rightarrow \infty}\left\langle\varphi, e^{i H t} \Phi_{r}(t) e^{-i H t} \varphi\right\rangle$ must be zero for each $\varphi \in \mathcal{H}$, and hence $\mathrm{w}-\lim _{t \rightarrow \infty} e^{i H t} \Phi_{r}(t) e^{-i H t}=0$ by polarization.

d) Let $f$ satisfy the hypothesis ii) and assume in addition that $f^{\prime}=g^{2}$ for some $g \in C_{c}^{\infty}(\mathbb{R})$. Let $\tilde{f} \in C_{c}^{\infty}(\mathbb{R})$ with support in $\left(c_{\eta}, \infty\right)$ be such that 
$\tilde{f} f^{\prime}=f^{\prime}$ and $0 \leq \tilde{f} \leq 1$. As in a), one finds that

$$
\begin{aligned}
\mathbf{D} \Phi_{r}(t)= & \frac{1}{t} \eta g\left\{\frac{1}{2 r} \tilde{\eta} P \cdot \frac{Q}{|Q|} \tilde{f}+\frac{1}{2 r} \tilde{f} \frac{Q}{|Q|} \cdot P \tilde{\eta}-\frac{|Q|}{2 r t}\right\} g \eta+O\left(t^{-2} r^{-2}\right) \\
& \leq-\left(c_{\eta}-\frac{c_{\eta}^{\prime \prime}}{r}\right) \frac{1}{t} \eta f^{\prime} \eta+O\left(t^{-2} r^{-2}\right) \leq O\left(t^{-2} r^{-2}\right) .
\end{aligned}
$$

Inserting this inequality in the formal identity

$$
e^{i H t} \Phi_{r}(t) e^{-i H t}=e^{i H t_{o}} \Phi_{r}\left(t_{o}\right) e^{-i H t_{o}}+\int_{t_{o}}^{t} e^{i H \tau} \mathbf{D} \Phi_{r}(\tau) e^{-i H \tau} d \tau
$$

with $t \geq t_{o} \geq 1$, one obtains the existence of some $c<\infty$ such that for all $\varphi \in \mathcal{H}^{2}$,

$$
0 \leq\left\langle\varphi, e^{i H t} \Phi_{r}(t) e^{-i H t} \varphi\right\rangle \leq\left\langle\varphi, e^{i H t_{o}} \Phi_{r}\left(t_{o}\right) e^{-i H t_{o}} \varphi\right\rangle+c\|\varphi\|^{2} t_{o}^{-1} r^{-2} .
$$

Both terms on the r.h.s. of (9) are independent of $t$ and tend to zero as $r$ increases. Since the existence of $\mathrm{s}-\lim _{t \rightarrow \infty} e^{i H t} \Phi_{r}(t) e^{-i H t}$ was shown in b), the inequalities in (9) imply that

$$
\mathrm{s}-\lim _{r \rightarrow \infty}\left(\mathrm{s}-\lim _{t \rightarrow \infty} e^{i H t} \Phi_{r}(t) e^{-i H t}\right)=0 .
$$

Now set $\Phi_{1}(t)=\Phi_{r}(t)+\tilde{\Phi}_{r}(t)$ with $\tilde{\Phi}_{r}(t):=\eta\left\{f\left(\frac{|Q|}{2 t}\right)-f\left(\frac{|Q|}{2 r t}\right)\right\} \eta$. Since the function $\{f(\cdot)-f(\dot{\bar{r}})\}$ has compact support in $\left(c_{\eta}, \infty\right)$ for any $r \geq 1$, the equation (8) is obtained by choosing $r$ large enough and by using (10) and the result of $\mathrm{c}$ ).

e) To prove ii) without assuming an additional condition for $f$, choose any real function $g \in C_{c}^{\infty}(\mathbb{R})$ such that supp $g \subset\left(c_{\eta}, \infty\right)$ and $\int_{-\infty}^{\infty} g^{2}(y) d y=1$, and set $F(x)=\int_{-\infty}^{x} g^{2}(y) d y$. If $f$ satisfies the hypothesis of ii), then $f-F$ satisfies that of i), and (8) follows by combining the results of c) and d).

In the next lemma, a certain distortion of the mapping $\mathbb{R}^{n} \ni x \mapsto \frac{1}{2} x^{2} \in \mathbb{R}$ will play a crucial technical role. We refer to [9] and especially to [4] for similar constructions used in the N-body problem. For $\delta>0$, let $m_{\delta} \in C^{\infty}(\mathbb{R})$ be a real convex function such that $m_{\delta}(y)=\frac{1}{2} \delta^{2}$ if $|y| \leq \delta$ and $m_{\delta}(y)=\frac{1}{2} y^{2}$ if $|y| \geq 2 \delta$. We set $r_{\delta}(x):=\sum_{j=1}^{n} m_{\delta}\left(x_{j}\right)$ for $x \in \mathbb{R}^{n}$. Observe that $r_{\delta}$ is a $C^{\infty}$-function on $\mathbb{R}^{n}$ with $\partial_{j k}^{2} r_{\delta}(x):=\frac{\partial^{2} r_{\delta}}{\partial x_{j} \partial x_{k}}(x)=0$ if $j \neq k$ and $\partial_{j j}^{2} r_{\delta}(x) \geq \xi^{2}\left(-\frac{x_{j}}{2 \delta}\right)+\xi^{2}\left(\frac{x_{j}}{2 \delta}\right)$ for any asymptotic localization function $\xi$ and all $x \in \mathbb{R}^{n}$.

Lemma 6.5. For each $\eta \in C_{c}^{\infty}(\mathbb{R})$, each $j \in\{1, \ldots, n\}$ and each $f \in$ $C_{c}^{\infty}\left(\mathbb{R}^{n}\right)$ with support in $Y_{j}^{ \pm}$, there exists a constant $c<\infty$ such that for all 
$\varphi \in \mathcal{H}$,

$$
\int_{1}^{\infty}\left\|f\left(\frac{Q}{2 t}\right)\left(P_{j}-\frac{Q_{j}}{2 t}\right) \eta(H) e^{-i H t} \varphi\right\|^{2} \frac{d t}{t} \leq c\|\varphi\|^{2} .
$$

Furthermore,

$$
s-\lim _{t \rightarrow \infty} f\left(\frac{Q}{2 t}\right)\left(P_{j}-\frac{Q_{j}}{2 t}\right) \eta(H) e^{-i H t}=0 .
$$

Proof. Let us fix a number $c_{\eta}^{\prime} \in \mathbb{R}$ such that $\operatorname{supp} \eta \subset\left(-\infty, c_{\eta}^{\prime}\right)$ and a function $\tilde{\eta} \in C_{c}^{\infty}\left(\left(-\infty, c_{\eta}^{\prime}\right)\right)$ satisfying $\tilde{\eta} \eta=\eta$ and $0 \leq \tilde{\eta} \leq 1$. Let $c_{\eta}$ be the positive constant depending on $\eta$ given by the previous lemma.

a) Consider $h \in C_{c}^{\infty}(\mathbb{R})$ such that $h(|x|) f(x)=f(x)$ for all $x \in \mathbb{R}^{n}$ and $h(y)=1$ if $y \in\left[0, c_{\eta}+1\right)$. For $\delta>0$ and $t \geq 1$, set

$$
L(t):=\frac{1}{2}\left(P-\frac{Q}{2 t}\right) \cdot \nabla r_{\delta}\left(\frac{Q}{2 t}\right)+\frac{1}{2} \nabla r_{\delta}\left(\frac{Q}{2 t}\right) \cdot\left(P-\frac{Q}{2 t}\right)+r_{\delta}\left(\frac{Q}{2 t}\right),
$$

and $\Phi(t):=\eta(H) h\left(\frac{|Q|}{2 t}\right) L(t) h\left(\frac{|Q|}{2 t}\right) \eta(H)$. One can check that $\Phi$ belongs to $B C_{H}^{1}([1, \infty), \mathcal{B}(\mathcal{H}))$ and that

$$
\begin{aligned}
\mathbf{D} \Phi(t)= & \eta\{\mathbf{D} h\} L h \eta+\eta h L\{\mathbf{D} h\} \eta-\eta h \nabla V(Q) \cdot \nabla r_{\delta} h \eta \\
& +\frac{1}{t} \eta h\left[P-\frac{Q}{2 t}\right]^{T} \partial^{2} r_{\delta}\left[P-\frac{Q}{2 t}\right] h \eta+O\left(t^{-3}\right),
\end{aligned}
$$

where $\partial^{2} r_{\delta}$ is the matrix of second derivatives of $r_{\delta}$. In order to be able to use Lemma 6.2, we obtain now some estimates for each term of (12).

Some commutator calculations, using repeatedly i) of Lemma 6.1, show that $\eta\{\mathbf{D} h\} L h \eta+\eta h L\{\mathbf{D} h\} \eta$ can be rewritten as $\frac{1}{t} \eta g\left(\frac{|Q|}{2 t}\right) \Psi(t) g\left(\frac{|Q|}{2 t}\right) \eta+$ $O\left(t^{-2}\right)$, where $\Psi$ is a bounded operator-valued mapping and $g$ belongs to $C_{c}^{\infty}(\mathbb{R})$ and satisfies the conditions supp $\subset\left(c_{\eta}, \infty\right)$ and $g(y) h^{\prime}(y)=h^{\prime}(y)$ for all $y \geq 0$. The statement i) of the previous lemma implies that the term $\frac{1}{t} \eta g \Psi g \eta$ is integrable along the evolution. We also observe that $h^{2} \nabla_{j} r_{\delta}$ belongs to $C_{c}^{\infty}\left(\mathbb{R}^{n}\right)$ and has its support in $Y_{j}^{-} \cup Y_{j}^{+}$. Therefore, using ii) of Lemma 6.1 , the term $\eta h \nabla V \cdot \nabla r_{\delta} h \eta$ belongs to $L^{1}((1, \infty), d t)$. Finally, the properties of $r_{\delta}$ mentioned before this lemma imply that

$$
\eta h\left[P-\frac{Q}{2 t}\right]^{T} \partial^{2} r_{\delta}\left[P-\frac{Q}{2 t}\right] h \eta \geq \eta h\left(P_{j}-\frac{Q_{j}}{2 t}\right) \xi^{2}\left( \pm \frac{Q_{j}}{4 \delta t}\right)\left(P_{j}-\frac{Q_{j}}{2 t}\right) h \eta
$$

for any asymptotic localization function $\xi$ and each $j \in\{1, \ldots, n\}$. Thus, by applying Lemma 6.2 with $B^{*}(t) B(t)=\frac{1}{t} \eta h\left(P_{j}-\frac{Q_{j}}{2 t}\right) \xi^{2}\left( \pm \frac{Q_{j}}{4 \delta t}\right)\left(P_{j}-\frac{Q_{j}}{2 t}\right) h \eta$, 
one obtains that there exists $c<\infty$ such that for each $j \in\{1, \ldots, n\}$ and all $\varphi \in \mathcal{H}$ :

$$
\int_{1}^{\infty}\left\|\xi\left( \pm \frac{Q_{j}}{4 \delta t}\right)\left(P_{j}-\frac{Q_{j}}{2 t}\right) h\left(\frac{|Q|}{2 t}\right) \eta(H) e^{-i H t} \varphi\right\|^{2} \frac{d t}{t} \leq c\|\varphi\|^{2} .
$$

Since $f$ has a compact support in $Y_{j}^{ \pm}$, we may now fix $j$ and $\delta$ such that $\xi\left( \pm \frac{x_{j}}{2 \delta}\right) f(x)=f(x)$ for all $x \in \mathbb{R}^{n}$. Then the first assertion of the lemma is a simple consequence of (13) and of the estimate $\left[P_{j}, h\left(\frac{|Q|}{2 t}\right)\right] \in O\left(t^{-1}\right)$.

b) For $t \geq 1$, set

$$
\Phi(t):=\eta(H)\left\{P_{j}-\frac{Q_{j}}{2 t}\right\} f\left(\frac{Q}{2 t}\right) \tilde{\eta}^{2}(H) f\left(\frac{Q}{2 t}\right)\left\{P_{j}-\frac{Q_{j}}{2 t}\right\} \eta(H),
$$

and notice that $\Phi$ belongs to $B C_{H}^{1}([1, \infty), \mathcal{B}(\mathcal{H}))$. One can check that

$$
\begin{aligned}
& \mathbf{D} \Phi(t)=-\frac{2}{t} \Phi(t)-\eta \nabla_{j} V f \tilde{\eta}^{2} f\left\{P_{j}-\frac{Q_{j}}{2 t}\right\} \eta-\eta\left\{P_{j}-\frac{Q_{j}}{2 t}\right\} f \tilde{\eta}^{2} f \nabla_{j} V \eta \\
& +\frac{1}{2 t} \eta\left\{P_{j}-\frac{Q_{j}}{2 t}\right\}\left\{\left(P-\frac{Q}{2 t}\right) \cdot \nabla f+\nabla f \cdot\left(P-\frac{Q}{2 t}\right)\right\} \tilde{\eta}^{2} f\left\{P_{j}-\frac{Q_{j}}{2 t}\right\} \eta \\
& +\frac{1}{2 t} \eta\left\{P_{j}-\frac{Q_{j}}{2 t}\right\} f \tilde{\eta}^{2}\left\{\left(P-\frac{Q}{2 t}\right) \cdot \nabla f+\nabla f \cdot\left(P-\frac{Q}{2 t}\right)\right\}\left\{P_{j}-\frac{Q_{j}}{2 t}\right\} \eta .
\end{aligned}
$$

Having in mind the use of Lemma 6.3 , we collect some estimates for each of these terms.

The second and the third terms on the r.h.s. belong to $L^{1}((1, \infty), d t)$ because $\eta \nabla_{j} V f \tilde{\eta}$ and $\tilde{\eta} f \nabla_{j} V \eta$ are integrable in norm and the remaining factors are norm bounded independently of $t$ for $t \geq 1$. Some further commutator calculations based on Lemma 6.1 show that the last two terms of the r.h.s. can be rewritten as $\frac{1}{2 t} \eta\left\{P_{j}-\frac{Q_{j}}{2 t}\right\} g\left(\frac{Q}{2 t}\right) \Psi(t) g\left(\frac{Q}{2 t}\right)\left\{P_{j}-\frac{Q_{j}}{2 t}\right\} \eta+O\left(t^{-2}\right)$, where $\Psi$ is a bounded operator-valued mapping and $g$ is a $C_{c}^{\infty}\left(\mathbb{R}^{n}\right)$-function with support in $Y_{j}^{ \pm}$and satisfying $g f=f$. One has now the estimate

$$
\begin{gathered}
\frac{1}{2 t}\left|\left\langle\psi, \eta\left\{P_{j}-\frac{Q_{j}}{2 t}\right\} g \Psi g\left\{P_{j}-\frac{Q_{j}}{2 t}\right\} \eta \varphi\right\rangle\right| \\
\leq \frac{c}{t}\left\|g\left\{P_{j}-\frac{Q_{j}}{2 t}\right\} \eta \psi\right\|\left\|g\left\{P_{j}-\frac{Q_{j}}{2 t}\right\} \eta \varphi\right\|
\end{gathered}
$$

for some positive constant $c<\infty$ and all $\psi, \varphi \in \mathcal{H}$.

Since the term $-\frac{2}{t} \Phi(t)$ satisfies a similar estimate, we can apply Lemma 6.3 with $E_{k}=F_{k}=\sqrt{\frac{c}{t}} g\left\{P_{j}-\frac{Q_{j}}{2 t}\right\} \eta$. By taking into account the first statement 
of the present lemma we obtain the existence of $\mathrm{s}-\lim _{t \rightarrow \infty} e^{i H t} \Phi(t) e^{-i H t}$. It follows that the $\operatorname{limit}_{t \rightarrow \infty}\left\|\tilde{\eta}(H) f\left(\frac{Q}{2 t}\right)\left(P_{j}-\frac{Q_{j}}{2 t}\right) \eta(H) e^{-i H t} \varphi\right\|$ exists for each $\varphi \in \mathcal{H}$. By commuting $\tilde{\eta}(H)$ to the right with the help of i) and iii) of Lemma 6.1, we get that $\lim _{t \rightarrow \infty}\left\|f\left(\frac{Q}{2 t}\right)\left(P_{j}-\frac{Q_{j}}{2 t}\right) \eta(H) e^{-i H t} \varphi\right\|$ exists for each $\varphi \in \mathcal{H}$. But this limit has to be equal to zero because of (11).

We now prove the existence of the asymptotic velocity. One major ingredient is a class of functions with some special property. Let $\mathfrak{F}$ be the set of all functions $f \in C_{c}^{\infty}\left(\mathbb{R}^{n}\right)$ such that, for each $j \in\{1, \ldots, n\}$, there exists a neighbourhood of the hypersurface $x_{j}=0$ in which $f$ does not depend on $x_{j}$. One can check that $\mathfrak{F}$ is dense in $C_{0}\left(\mathbb{R}^{n}\right)$.

Lemma 6.6. For each $f \in C_{0}\left(\mathbb{R}^{n}\right)$, the following limit exists:

$$
s-\lim _{t \rightarrow \infty} e^{i H t} f\left(\frac{Q}{2 t}\right) e^{-i H t} .
$$

Proof. By density in $\mathcal{H}$ of the set of vectors of the form $\eta^{2}(H) \varphi$ with $\eta \in$ $C_{c}^{\infty}(\mathbb{R})$ and $\varphi \in \mathcal{H}$, it is enough to prove that $\mathrm{s}-\lim _{t \rightarrow \infty} e^{i H t} f\left(\frac{Q}{2 t}\right) e^{-i H t} \eta^{2}(H)$ exists, which is equivalent by i) of Lemma 6.1 with the existence of

$$
\mathrm{s}-\lim _{t \rightarrow \infty} \eta(H) e^{i H t} f\left(\frac{Q}{2 t}\right) e^{-i H t} \eta(H)
$$

Since $\mathfrak{F}$ is dense in $C_{0}\left(\mathbb{R}^{n}\right)$, there is also no loss of generality in assuming that $f \in \mathfrak{F}$. For $t \geq 1$, set

$$
\Phi(t):=\eta\left\{\frac{1}{2}\left(P-\frac{Q}{2 t}\right) \cdot \nabla f\left(\frac{Q}{2 t}\right)+\frac{1}{2} \nabla f\left(\frac{Q}{2 t}\right) \cdot\left(P-\frac{Q}{2 t}\right)+f\left(\frac{Q}{2 t}\right)\right\} \eta .
$$

One observes that $\Phi \in B C_{H}^{1}([1, \infty), \mathcal{B}(\mathcal{H}))$ and that, similarly to $(12)$,

$$
\mathbf{D} \Phi(t)=-\eta \nabla f \cdot \nabla V(Q) \eta+\frac{1}{t} \eta\left[P-\frac{Q}{2 t}\right]^{T} \partial^{2} f\left[P-\frac{Q}{2 t}\right] \eta+O\left(t^{-3}\right) .
$$

For each $j \in\{1, \ldots, n\}, \nabla_{j} f$ belongs to $C_{c}^{\infty}\left(\mathbb{R}^{n}\right)$ and has its support in $Y_{j}^{-} \cup$ $Y_{j}^{+}$. Thus by ii) of Lemma 6.1 the first term on the r.h.s. of (15) belongs to $L^{1}((1, \infty), d t)$. The second term is equal to

$$
\sum_{j, k=1}^{n} \frac{1}{t} \eta\left(P_{j}-\frac{Q_{j}}{2 t}\right) g_{j}\left(\frac{Q}{2 t}\right)\left\{\partial_{j k}^{2} f\right\} g_{k}\left(\frac{Q}{2 t}\right)\left(P_{k}-\frac{Q_{k}}{2 t}\right) \eta,
$$


where each $g_{j}$ belongs to $C_{c}^{\infty}\left(\mathbb{R}^{n}\right)$, has support in $Y_{j}^{-} \cup Y_{j}^{+}$and satisfies $g_{j} \nabla_{j} f=\nabla_{j} f$. By applying Lemma 6.3 and by using the first statement of Lemma 6.5, one obtains the existence of $\mathrm{s}-\lim _{t \rightarrow \infty} e^{i H t} \Phi(t) e^{-i H t}$. But the second assertion of Lemma 6.5 implies that this limit is equal to (14), which therefore exists.

Assume for a while that $f$ is a complex function belonging to $C_{0}\left(\mathbb{R}^{n}\right)$. By writing $f=f_{1}+i f_{2}$ with $f_{1}, f_{2}$ two real $C_{0}\left(\mathbb{R}^{n}\right)$-functions, one gets from the previous lemma the existence of $\mathrm{s}-\lim _{t \rightarrow \infty} e^{i H t} f\left(\frac{Q}{2 t}\right) e^{-i H t}$, which we denote by $\mathfrak{P}(f)$. Since $\mathfrak{P}(f g)=\mathfrak{P}(f) \mathfrak{P}(g)$ and $\mathfrak{P}(\bar{f})=\mathfrak{P}(f)^{*}$ for two complex functions $f, g \in C_{0}\left(\mathbb{R}^{n}\right)$, the mapping $\mathfrak{P}: C_{0}\left(\mathbb{R}^{n}\right) \rightarrow \mathcal{B}(\mathcal{H})$ is a morphism between two $\mathrm{C}^{*}$-algebras. Let $B O\left(\mathbb{R}^{n}\right)$ be the unital $\mathrm{C}^{*}$-algebra of bounded Borel functions on $\mathbb{R}^{n}$. We say that a sequence $\left\{f_{k}\right\}_{k \in \mathbb{N}}$ in $C_{0}\left(\mathbb{R}^{n}\right)$ is bound-

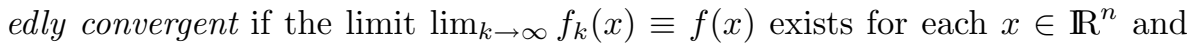
$\left|f_{k}(x)\right| \leq c$ for some constant $c<\infty$ independent of $k$ and $x$ ( $f$ belongs then to $B O\left(\mathbb{R}^{n}\right)$, cf. [1], Section 8.1.1). It is also proved in this reference that the morphism $\mathfrak{P}$ has a unique extension to a morphism $\widetilde{\mathfrak{P}}: B O\left(\mathbb{R}^{n}\right) \rightarrow \mathcal{B}(\mathcal{H})$ such that $\mathrm{s}-\lim _{k \rightarrow \infty} \widetilde{\mathfrak{P}}\left(f_{k}\right)=\widetilde{\mathfrak{P}}(f)$ if $\left\{f_{k}\right\}_{k \in \mathbb{N}}$ converges boundedly to $f$.

Let $\chi_{\Xi}$ denote the characteristic function of the Borel set $\Xi \subset \mathbb{R}^{n}$ and $\mathbb{I}_{\Xi}:=\widetilde{\mathfrak{P}}\left(\chi_{\Xi}\right)$. Then $\mathbb{I}: \mathbb{R}^{n} \supset \Xi \mapsto \mathbb{I}_{\Xi} \in \mathcal{B}(\mathcal{H})$ determines a projection-valued measure on $\mathbb{R}^{n}$. In the next lemma we shall prove that $\mathbb{I}_{\mathbb{R}^{n}}$ is the identity operator in $\mathcal{B}(\mathcal{H})$, and therefore $\mathbb{I}$ becomes a spectral measure on $\mathbb{R}^{n}$. So, if $\mathcal{P}:=\int_{\mathbb{R}^{n}} x \mathbb{I}(d x)$ is the $n$-tuple of commuting self-adjoint operators in $\mathcal{H}$ determined by $\mathbb{I}$, then clearly $\mathfrak{P}(f)=f(\mathcal{P})$. The $n$-tuple $\mathcal{P}$ is commonly called the asymptotic velocity.

Lemma 6.7. $\quad$ i) The projection-valued measure $\mathbb{I}$ satisfies the relation $\mathbb{I}_{\mathbb{R}^{n}}=I$.

ii) For each $\eta \in C_{0}(\mathbb{R})$ and each $f \in C_{0}\left(\mathbb{R}^{n}\right)$ one has $[\eta(H), f(\mathcal{P})]=0$, i.e. the asymptotic velocity $\mathcal{P}$ commutes with the Hamiltonian $H$.

Proof. a) Let $f \in C_{c}^{\infty}(\mathbb{R})$ be such that $f=1$ in a neighbourhood of 0 . Then, since $\left\{f\left(\frac{|\cdot|}{r}\right)\right\}_{r \geq 1}$ converges boundedly to the function $\mathbb{R}^{n} \ni x \mapsto 1$, the relation $\mathbb{I}_{\mathbb{R}^{n}}=I$ is satisfied if $\mathrm{s}-\lim _{r \rightarrow \infty} \mathrm{s}-\lim _{t \rightarrow \infty} e^{i H t} f\left(\frac{|Q|}{2 r t}\right) e^{-i H t}=I$.

Let $\eta \in C_{c}^{\infty}(\mathbb{R})$ and $r \geq 1$. Using i) of Lemma 6.1 , one observes that $\mathrm{s}-\lim _{t \rightarrow \infty}\left\{I-e^{i H t} f\left(\frac{|Q|}{2 r t}\right) e^{-i H t}\right\} \eta^{2}=\mathrm{s}-\lim _{t \rightarrow \infty} e^{i H t} \eta\left\{I-f\left(\frac{|Q|}{2 r t}\right)\right\} \eta e^{-i H t}$. 
The r.h.s. is zero for $r$ large enough (depending on $\eta$ ) by the second assertion of Lemma 6.4. Hence $\mathrm{s}-\lim _{r \rightarrow \infty} \mathrm{s}-\lim _{t \rightarrow \infty}\left\{I-e^{i H t} f\left(\frac{|Q|}{2 r t}\right) e^{-i H t}\right\} \eta^{2}(H)=0$. Since the set of vectors of the form $\eta^{2}(H) \varphi$ with $\eta \in C_{c}^{\infty}(\mathbb{R})$ and $\varphi \in \mathcal{H}$ is dense in $\mathcal{H}$, this finishes the proof of i).

b) The proof that $[\eta(H), f(\mathcal{P})]=0$ for each $\eta \in C_{c}^{\infty}(\mathbb{R})$ and each $f \in$ $C_{c}^{\infty}\left(\mathbb{R}^{n}\right)$ is easily obtained with the help of the statement i) of Lemma 6.1. The assertion ii) follows then by density of $C_{c}^{\infty}\left(\mathbb{R}^{n}\right)$ in $C_{0}\left(\mathbb{R}^{n}\right)$ and density of $C_{c}^{\infty}(\mathbb{R})$ in $C_{0}(\mathbb{R})$.

The next statement is usually called minimal velocity estimate. It is a nontrivial consequence of the Mourre estimate obtained in Section 5 . We point out that the $C_{u}^{1}(A)$-condition of Proposition 6.1 is required in order to fulfil the hypotheses of Proposition 5.2. Let us recall that $\kappa(H)$ is a closed countable set which contains the thresholds and the eigenvalues of $H$.

Lemma 6.8. For each $\eta \in C_{c}^{\infty}(\mathbb{R} \backslash \kappa(H))$, there exists $\varepsilon_{\eta}>0$ such that for some $c<\infty$ and all $\varphi \in \mathcal{H}$,

$$
\int_{1}^{\infty}\left\|\chi_{\left[0, \varepsilon_{\eta}\right]}\left(\frac{|Q|}{2 t}\right) \eta(H) e^{-i H t} \varphi\right\|^{2} \frac{d t}{t} \leq c\|\varphi\|^{2} .
$$

Proof. Let $\tilde{\eta} \in C_{c}^{\infty}(\mathbb{R} \backslash \kappa(H))$ be such that $\tilde{\eta} \eta=\eta$ and $0 \leq \tilde{\eta} \leq 1$. Let $\theta:=\min _{\lambda} \varrho_{H}^{A}(\lambda)$ for $\lambda \in \operatorname{supp} \tilde{\eta}$. It follows from Propositions 5.1 and 5.2 that $\theta$ is strictly positive and that $\tilde{\eta}(H)[i H, A] \tilde{\eta}(H) \geq \theta \tilde{\eta}^{2}(H)$.

We now fix $\varepsilon_{\eta}$ and $\varepsilon_{\eta}^{\prime}$ such that $0<\varepsilon_{\eta}<\varepsilon_{\eta}^{\prime}<\frac{\theta}{2 n}\left(\sup _{j}\left\|\tilde{\eta}(H) P_{j}\right\|\right)^{-1}$. The case $\tilde{\eta}(H)=0$ is excluded because the statement is then trivial. If $B(0, \delta)$ denotes the open ball in $\mathbb{R}^{n}$ of center 0 and radius $\delta>0$, let $f \in \mathfrak{F}$ be such that $\operatorname{supp} f \subset B\left(0, \varepsilon_{\eta}^{\prime}\right)$ and $f=1$ on $B\left(0, \varepsilon_{\eta}\right)$. Let also $\tilde{f} \in C_{c}^{\infty}\left(\mathbb{R}^{n}\right)$ with support in $B\left(0, \varepsilon_{\eta}^{\prime}\right)$ be such that $\tilde{f} f=f$ and $0 \leq \tilde{f} \leq 1$. For $t \geq 1$, set $M(t):=\left(P-\frac{Q}{2 t}\right) \cdot \nabla f\left(\frac{Q}{2 t}\right)+f\left(\frac{Q}{2 t}\right)$ and $\Phi(t):=\eta(H) M \tilde{\eta}(H) \frac{A}{t} \tilde{\eta}(H) M^{*} \eta(H)$. The statement v) of Lemma 6.1 assures that $\Phi$ is a bounded operator-valued mapping. Moreover, one can easily check that $\Phi$ is differentiable in norm with bounded derivative. It follows then that $\Phi \in B C_{H}^{1}([1, \infty), \mathcal{B}(\mathcal{H}))$. So, let us calculate the Heisenberg derivative of $\Phi$ :

$$
\begin{aligned}
\mathbf{D} \Phi(t)= & -\eta \nabla f \cdot \nabla V \tilde{\eta} \frac{A}{t} \tilde{\eta} M^{*} \eta-\eta M \tilde{\eta} \frac{A}{t} \tilde{\eta} \nabla f \cdot \nabla V \eta \\
& +\frac{1}{t} \eta\left[P-\frac{Q}{2 t}\right]^{T} \partial^{2} f\left[P-\frac{Q}{2 t}\right] \tilde{\eta} \frac{A}{t} \tilde{\eta} M^{*} \eta+\mathrm{hc} \\
& +\eta M \tilde{\eta}\left\{\left[i H, \frac{A}{t}\right]-\frac{A}{t^{2}}\right\} \tilde{\eta} M^{*} \eta+O\left(t^{-2}\right) .
\end{aligned}
$$


In order to be able to use Lemma 6.2 we obtain now some estimates for each of these terms.

Since $f \in \mathfrak{F}$, the statement ii) of Lemma 6.1 implies that the first two terms on the r.h.s. of $(16)$ belong to $L^{1}((1, \infty), d t)$. Some further commutator calculations (based on Lemma 6.1) show that the third and the fourth terms on the r.h.s. of (16) can be rewritten as

$$
\begin{gathered}
\sum_{j, k=1}^{n} \frac{1}{t} \eta\left(P_{j}-\frac{Q_{j}}{2 t}\right) g_{j}\left(\frac{Q}{2 t}\right) \Psi_{j k}(t) g_{k}\left(\frac{Q}{2 t}\right)\left(P_{k}-\frac{Q_{k}}{2 t}\right) \eta \\
+O\left(t^{-2}\right)+\frac{1}{t} L^{1}((1, \infty), d t)
\end{gathered}
$$

where $\Psi_{j k}=\left(\partial_{j k}^{2} f\right) \tilde{\eta} \frac{A}{t} \tilde{\eta} M^{*} \tilde{\eta}+\tilde{\eta} M \tilde{\eta} \frac{A}{t} \tilde{\eta}\left(\partial_{j k}^{2} f\right)$ is a bounded operator-valued mapping and each $g_{j}$ belongs to $C_{c}^{\infty}\left(\mathbb{R}^{n}\right)$, has its support in $Y_{j}^{-} \cup Y_{j}^{+}$and satisfies $g_{j} \nabla_{j} f=\nabla_{j} f$. By taking Lemma 6.5 into account, one finds that the first term of (17) is integrable along the evolution.

For the fifth term on the r.h.s. of (16) one can check that

$$
\eta M \tilde{\eta}\left[i H, \frac{A}{t}\right] \tilde{\eta} M^{*} \eta \geq \frac{\theta}{t} \eta M \tilde{\eta}^{2} M^{*} \eta=\frac{\theta}{t} \eta M M^{*} \eta+O\left(t^{-2}\right)+\frac{1}{t} L^{1}((1, \infty), d t),
$$

where the equality has been obtained with the help of i) and iii) of Lemma 6.1. Furthermore, since $A=\frac{1}{4}(P \cdot Q+Q \cdot P)$, one finds that (by commuting the first $\tilde{f}$ to the right for the second inequality)

$$
\begin{aligned}
\left\|\tilde{f} \tilde{\eta} \frac{A}{t} \tilde{\eta} \tilde{f}\right\| & \leq\left\|\tilde{f} \tilde{\eta} P \cdot \frac{Q}{2 t} \tilde{\eta} \tilde{f}\right\|+O\left(t^{-1}\right) \leq n \sup _{j}\left\{\left\|\tilde{\eta} P_{j}\right\|\left\|\tilde{f} \frac{Q_{j}}{2 t}\right\|\right\}+O\left(t^{-1}\right) \\
& \leq n \varepsilon_{\eta}^{\prime} \sup _{j}\left\|\tilde{\eta} P_{j}\right\|+O\left(t^{-1}\right),
\end{aligned}
$$

which is less than $\frac{\theta}{2}+O\left(t^{-1}\right)$. It follows that

$$
-\frac{1}{t} \eta M \tilde{f} \tilde{\eta} \frac{A}{t} \tilde{\eta} \tilde{f} M^{*} \eta \geq-\frac{\theta}{2 t} \eta M M^{*} \eta+O\left(t^{-2}\right)
$$

and hence one gets that:

(18) $\eta M \tilde{\eta}\left\{\left[i H, \frac{A}{t}\right]-\frac{A}{t^{2}}\right\} \tilde{\eta} M^{*} \eta \geq \frac{\theta}{2 t} \eta M M^{*} \eta+O\left(t^{-2}\right)+\frac{1}{t} L^{1}((1, \infty), d t)$.

From the inequality $(a+b)(a+b)^{*} \geq(1-\nu) a a^{*}+\left(1-\frac{1}{\nu}\right) b b^{*}$, valid for any $\nu$ with $0<\nu<1$ one deduces that (take $\nu=\frac{1}{2}$ )

$$
\eta M M^{*} \eta \geq \frac{1}{2} \eta f^{2} \eta-\eta\left\{P-\frac{Q}{2 t}\right\} \cdot \nabla f \nabla f \cdot\left\{P-\frac{Q}{2 t}\right\} \eta .
$$


One can check with Lemma 6.5 that the term $\frac{1}{t} \eta\left\{P-\frac{Q}{2 t}\right\} \cdot \nabla f \nabla f \cdot\left\{P-\frac{Q}{2 t}\right\} \eta$ is integrable along the evolution. Thus by inserting (19) into (18), by applying then Lemma 6.2 with the term $B^{*}(t) B(t)$ equal to $\frac{\theta}{4 t} \eta f^{2} \eta$ and by taking into account all previous estimates, one obtains the expected result.

In the next lemma, we prove that the subspace of $\mathcal{H}$ of the states with zero asymptotic velocity is equal to the subspace spanned by the eigenvectors of $H$.

Lemma 6.9. The range of $E_{\{0\}}(\mathcal{P})$ is equal to the range of $E_{\mathrm{p}}(H)$.

Proof. First, let $\varphi \in \mathcal{H}$ such that $H \varphi=\lambda \varphi$. For each $f \in C_{0}\left(\mathbb{R}^{n}\right)$ and $t \geq 1$, one has

$$
\begin{aligned}
\left\|e^{i H t} f\left(\frac{Q}{2 t}\right) e^{-i H t} \varphi-f(0) \varphi\right\| & =\left\|e^{i(H-\lambda) t} f\left(\frac{Q}{2 t}\right) \varphi-f(0) \varphi\right\| \\
& =\left\|\left(f\left(\frac{Q}{2 t}\right)-f(0)\right) \varphi\right\| .
\end{aligned}
$$

The r.h.s. tends to zero as $t$ increases, and therefore $f(\mathcal{P}) \varphi=f(0) \varphi$, or equivalently $\varphi \in E_{\{0\}}(\mathcal{P}) \mathcal{H}$. Since $E_{\mathrm{p}}(H)$ and $E_{\{0\}}(\mathcal{P})$ are closed subspaces, it follows that any $\varphi \in E_{\mathrm{p}}(H) \mathcal{H}$ belongs to $E_{\{0\}}(\mathcal{P}) \mathcal{H}$.

Let us now show that $E_{\{0\}}(\mathcal{P}) \mathcal{H}$ is orthogonal to the continuous subspace of $\mathcal{H}$ with respect to $H$. So let $\varphi \in E_{\{0\}}(\mathcal{P}) \mathcal{H}$, i.e. $f(\mathcal{P}) \varphi=f(0) \varphi$ for each $f \in$ $C_{0}\left(\mathbb{R}^{n}\right)$. It is enough to prove that $\langle\psi, \varphi\rangle=0$ for any $\psi$ satisfying $\eta(H) \psi=\psi$ with $\eta \in C_{c}^{\infty}(\mathbb{R} \backslash \kappa(H))$. Let $\varepsilon_{\eta}$ be given by the previous lemma, and let $f \in C_{c}^{\infty}\left(\mathbb{R}^{n}\right)$ with support in $B\left(0, \varepsilon_{\eta}\right)$ be such that $f(0) \neq 0$. We observe that $\mathrm{s}-\lim _{t \rightarrow \infty} \eta e^{i H t} f^{2}\left(\frac{Q}{2 t}\right) e^{-i H t} \eta=0$ since the limit exists and since there exists $c<\infty$ such that $\int_{1}^{\infty}\left\|f\left(\frac{Q}{2 t}\right) \eta(H) e^{-i H t} v\right\|^{2} \frac{d t}{t} \leq c\|v\|^{2}$ for all $v \in \mathcal{H}$. We finally have

$$
\langle\psi, \varphi\rangle=\frac{1}{f^{2}(0)}\left\langle\eta \psi, f^{2}(\mathcal{P}) \eta \varphi\right\rangle=\frac{1}{f^{2}(0)} \lim _{t \rightarrow \infty}\left\langle\eta e^{i H t} f^{2}\left(\frac{Q}{2 t}\right) e^{-i H t} \eta \psi, \varphi\right\rangle=0 .
$$

\section{$\S 7 . \quad$ Asymptotic Completeness}

In this last section, we prove the existence of some suitably defined wave operators and, as a by-product, obtain the asymptotic completeness. For this 
purpose, we assume firstly that $V$ is a cartesian potential such that $H=-\boldsymbol{\Delta}+V$ is of class $C_{u}^{1}(A)$, and secondly that $V$ satisfies the requirement

$$
\int_{0}^{\infty}\left\|\xi\left((\beta-\alpha) \cdot \frac{Q}{r}\right)\left(V^{\alpha}(Q)-V^{\beta}(Q)\right)\right\|_{\mathcal{H}_{\alpha}^{2} \rightarrow \mathcal{H}_{\alpha}} d r<\infty
$$

for each $\alpha \in \mathcal{L}$, each $\beta \lessdot \alpha$ and some asymptotic localization function $\xi$. This last assumption means that each function $V^{\alpha}$ tends to its asymptotic limits in a short-range way.

Let us show that the above hypotheses are sufficient for the existence of the asymptotic velocity for each operator $H^{\alpha}$. Indeed, let $\alpha, \beta \in \mathcal{L}$ and $j \in\{1, \ldots, n\}$ be such that $\beta \lessdot \alpha, \alpha_{j}=0$ and $\beta_{j}= \pm 1$. In such a situation $V^{\beta}$ does not depend on $x_{j}$, and thus $\nabla_{j} V^{\alpha}(Q)$ is formally equal to $i P_{j}\left(V^{\alpha}(Q)-V^{\beta}(Q)\right)-i\left(V^{\alpha}(Q)-V^{\beta}(Q)\right) P_{j}$. Then some simple calculations, using this observation and the assumption (20), show that for each $j$ such that $\alpha_{j}=0$ :

$$
\int_{0}^{\infty}\left\|\xi\left( \pm \frac{Q_{j}}{r}\right) \nabla_{j} V^{\alpha}(Q)\right\|_{\mathcal{H}_{\alpha}^{2} \rightarrow \mathcal{H}_{\alpha}^{-2}} d r<\infty .
$$

Let us notice that in the special case $\alpha=o,(21)$ is nothing but (5). So, for each $\alpha \in \mathcal{L}, V^{\alpha}$ is a cartesian potential relative to $\mathcal{L}^{\alpha}$ which satisfies $(21)$ and such that $H^{\alpha}=-\boldsymbol{\Delta}^{\alpha}+V^{\alpha}$ is of class $C_{u}^{1}\left(A^{\alpha}\right)$ ( $c f$. Lemma 5.2). A proposition similar to Proposition 6.1 can therefore be stated for $H^{\alpha}$, with the only difference that the corresponding asymptotic velocity $\mathcal{P}^{\alpha}$ is a $|\alpha|$-tuple instead of a $n$-tuple. For the case $\alpha=o$, we keep the notation $\mathcal{P}$ instead of $\mathcal{P}^{o}$.

Moreover, a direct consequence of Lemma 9.4 .8 of [1] is that if the requirement (20) is assumed, then this condition is also satisfied with the norm $\|\cdot\|_{\mathcal{H}^{2} \rightarrow \mathcal{H}}$ instead of the norm $\|\cdot\|_{\mathcal{H}_{\alpha}^{2} \rightarrow \mathcal{H}_{\alpha}}$. It follows that (21) is fulfilled with the norm $\|\cdot\|_{\mathcal{H}^{2} \rightarrow \mathcal{H}^{-2}}$ instead of the norm $\|\cdot\|_{\mathcal{H}_{\alpha}^{2} \rightarrow \mathcal{H}_{\alpha}^{-2}}$. We notice furthermore that (21) is trivially satisfied for $k$ such that $\alpha_{k} \neq 0$ (since $V^{\alpha}$ does not depend on the variable $x_{k}$ ) and that $V^{\alpha}$ is a cartesian potential relative to $\mathcal{L}$. Hence, since $H_{\alpha}=-\boldsymbol{\Delta}+V^{\alpha}$ is of class $C_{u}^{1}(A)$, the hypotheses of Proposition 6.1 are fulfilled with $H_{\alpha}$ instead of $H$, and all developments of the previous section can be rewritten in terms of $H_{\alpha}$. Let $\mathcal{P}_{\alpha}$ denote the asymptotic velocity obtained for the operator $H_{\alpha}$. The relation between $\mathcal{P}_{\alpha}$ and $\mathcal{P}^{\alpha}$ will be given later on.

Let us mention that there exist situations for which the condition (20) can easily be checked.

Example 4. If $V$ is the sum of a Mourre potential $V_{m}$ constructed in Example 2 and of a short-range potential $V_{\mathrm{SR}}$ satisfying the additional condition 
$\lim _{r \rightarrow \infty}\left\|\xi\left(\frac{|Q|}{r}\right) V_{\mathrm{SR}}(Q)\right\|_{\mathcal{H}^{2} \rightarrow \mathcal{H}}=0$ for some asymptotic localization function $\xi$, then (20) is automatically satisfied. A similar conclusion is obtained if in Example 3 each $V_{j}$ reaches its limits in a short-range way. In both situations all hypotheses of Theorems 1.1 and 1.2 are fulfilled.

Proposition 7.1. Let $V$ be a cartesian potential such that $H=-\boldsymbol{\Delta}+V$ is of class $C_{u}^{1}(A)$. Assume moreover that the condition (20) is satisfied. Then for each $\alpha \in \mathcal{L}$, the following wave operators exist:

$$
\begin{aligned}
& W_{\alpha o}^{+}:=s-\lim _{t \rightarrow+\infty} e^{i H_{\alpha} t} e^{-i H t} E_{Y_{\alpha}}(\mathcal{P}), \\
& W_{o \alpha}^{+}:=s-\lim _{t \rightarrow+\infty} e^{i H t} e^{-i H_{\alpha} t} E_{Y_{\alpha}}\left(\mathcal{P}_{\alpha}\right) .
\end{aligned}
$$

These operators satisfy the relations

$$
W_{o \alpha}^{+} E_{\Xi}\left(\mathcal{P}_{\alpha}\right)=E_{\Xi}(\mathcal{P}) W_{o \alpha}^{+} \quad \text { and } \quad W_{\alpha o}^{+} E_{\Xi}(\mathcal{P})=E_{\Xi}\left(\mathcal{P}_{\alpha}\right) W_{\alpha o}^{+}
$$

for any Borel subset $\Xi$ of $Y_{\alpha}$.

Proof. In order to prove the existence of $W_{\alpha o}^{+}$, it is enough to show that for each $\eta \in C_{c}^{\infty}(\mathbb{R})$ and each $f$ in a dense subset of all $C_{0}\left(\mathbb{R}^{n}\right)$-functions with support in $Y_{\alpha}$, the limit $\mathrm{s}-\lim _{t \rightarrow \infty} e^{i H_{\alpha} t} e^{-i H t} f(\mathcal{P}) \eta^{2}(H)$ exists.

a) Let $f \in \mathfrak{F}$ be such that $\operatorname{supp} f \subset Y_{\alpha}$. For $t \geq 1$, set $M(t):=f\left(\frac{Q}{2 t}\right)+$ $\nabla f\left(\frac{Q}{2 t}\right) \cdot\left(P-\frac{Q}{2 t}\right)$ and $\Phi(t):=\eta\left(H_{\alpha}\right) M(t) \eta(H)$. One can check that $\Phi$ belongs to $B C^{1}([1, \infty), \mathcal{B}(\mathcal{H}))$. Let us assume for a while that the limit $\mathrm{s}-$ $\lim _{t \rightarrow \infty} e^{i H_{\alpha} t} \Phi(t) e^{-i H t}$ exists. Then one observes that (in the strong topology)

$$
\begin{aligned}
& \lim _{t \rightarrow \infty} e^{i H_{\alpha} t} \eta\left(H_{\alpha}\right) M(t) \eta(H) e^{-i H t}=\lim _{t \rightarrow \infty} e^{i H_{\alpha} t} \eta(H) M(t) \eta(H) e^{-i H t} \\
& =\lim _{t \rightarrow \infty} e^{i H_{\alpha} t} \eta(H) f\left(\frac{Q}{2 t}\right) \eta(H) e^{-i H t}=\lim _{t \rightarrow \infty} e^{i H_{\alpha} t} e^{-i H t} f(\mathcal{P}) \eta^{2}(H),
\end{aligned}
$$

where we have used successively iv) of Lemma 6.1, the second assertion of Lemma 6.5 and the hypothesis $f \in \mathfrak{F}$, and finally the existence of the asymptotic velocity $\mathcal{P}$. Thus the existence of $\mathrm{s}-\lim _{t \rightarrow \infty} e^{i H_{\alpha} t} e^{-i H t} f(\mathcal{P}) \eta^{2}(H)$ follows from the existence of $\mathrm{s}-\lim _{t \rightarrow \infty} e^{i H_{\alpha} t} \Phi(t) e^{-i H t}$, and the latter will be established below with the help of Lemma 6.3.

b) One can check that

$$
\begin{aligned}
& i H_{\alpha} \Phi(t)-i \Phi(t) H+\frac{d}{d t} \Phi(t)=-i \eta\left(H_{\alpha}\right)\left(V(Q)-V^{\alpha}(Q)\right) M \eta(H) \\
& +\eta\left(H_{\alpha}\right)\left\{-\nabla f \cdot \nabla V+\frac{1}{t}\left[P-\frac{Q}{2 t}\right]^{T} \partial^{2} f\left[P-\frac{Q}{2 t}\right]\right\} \eta(H)+O\left(t^{-2}\right) .
\end{aligned}
$$


Since $\nabla_{j} f$ has support in $Y_{j}^{-} \cup Y_{j}^{+}$, the term $\eta\left(H_{\alpha}\right) \nabla f \cdot \nabla V \eta(H)$ belongs to $L^{1}((1, \infty), d t)$ (the proof of this fact is similar to that of the statement ii) of Lemma 6.1). One observes that the term $\frac{1}{t} \eta\left(H_{\alpha}\right)\left[P-\frac{Q}{2 t}\right]^{T} \partial^{2} f\left[P-\frac{Q}{2 t}\right] \eta(H)$ in (23) is equal to

$$
\sum_{j, k=1}^{n} \frac{1}{t} \eta\left(H_{\alpha}\right)\left(P_{j}-\frac{Q_{j}}{2 t}\right) g_{j}\left(\frac{Q}{2 t}\right)\left\{\partial_{j k}^{2} f\right\} g_{k}\left(\frac{Q}{2 t}\right)\left(P_{k}-\frac{Q_{k}}{2 t}\right) \eta(H),
$$

where each $g_{j}$ belongs to $C_{c}^{\infty}\left(\mathbb{R}^{n}\right)$, has support in $Y_{j}^{-} \cup Y_{j}^{+}$and satisfies $g_{j} \nabla_{j} f=\nabla_{j} f$. In relation with (24), it is useful to recall (from Lemma 6.5) that for each $k \in\{1, \ldots, n\}$, there exists $c<\infty$ such that

$$
\int_{1}^{\infty}\left\|g_{k}\left(\frac{Q}{2 t}\right)\left(P_{k}-\frac{Q_{k}}{2 t}\right) \eta(H) e^{-i H t} \varphi\right\|^{2} \frac{d t}{t} \leq c\|\varphi\|^{2}
$$

for all $\varphi \in \mathcal{H}$, and that a similar result can be obtained with $H_{\alpha}$ instead of $H$ (see the discussion before Example 4).

We finally notice that $\int_{1}^{\infty}\left\|\prod_{\left\{j \mid \alpha_{j} \neq 0\right\}} \xi\left(\alpha_{j} \frac{Q_{j}}{\delta r}\right)\left(V-V^{\alpha}\right)\right\|_{\mathcal{H}^{2} \rightarrow \mathcal{H}} d r<\infty$ for each $\delta>0$. This follows directly from (20) and Lemma 9.4.8 of [1] by inserting and removing suitable terms between $V$ and $V^{\alpha}$. Then by choosing $\delta$ such that $\prod_{\left\{j \mid \alpha_{j} \neq 0\right\}} \xi\left(\alpha_{j} \frac{x_{j}}{\delta}\right) f(x)=f(x)$ for all $x \in \mathbb{R}^{n}$, one can easily show that $\eta\left(H_{\alpha}\right)\left(V-V^{\alpha}\right) M \eta(H)$ belongs to $L^{1}((1, \infty), d t)$.

In view of the preceding estimates, the existence of

$$
\mathrm{s}-\lim _{t \rightarrow \infty} e^{i H_{\alpha} t} \Phi(t) e^{-i H t}
$$

is seen to be a direct consequence of Lemma 6.3.

c) By similar arguments, we can show the existence of $W_{o \alpha}^{+}$. For the last assertion of the proposition, one easily observes that $W_{\text {o }}^{+} f\left(\mathcal{P}_{\alpha}\right)=f(\mathcal{P}) W_{o \alpha}^{+}$ and that $W_{\alpha o}^{+} f(\mathcal{P})=f\left(\mathcal{P}_{\alpha}\right) W_{\alpha o}^{+}$for each $f \in C_{0}\left(\mathbb{R}^{n}\right)$ with support in $Y_{\alpha}$. The conclusion follows then by taking a sequence $\left\{f_{k}\right\}_{k \in \mathbb{N}}$ of functions in $C_{0}\left(\mathbb{R}^{n}\right)$ with $\operatorname{supp} f_{k} \subset Y_{\alpha}$ such that this sequence converges boundedly to $\chi_{\Xi}$.

Proof of Theorem 1.2. Since $Z_{\alpha}$ is a subset of $Y_{\alpha}$, the existence of $\Omega_{\alpha}^{+}$ follows from Proposition 7.1. One gets from the relations (22) that

$$
\operatorname{Ran}\left(W_{o \alpha}^{+} E_{z_{\alpha}}\left(\mathcal{P}_{\alpha}\right)\right) \subset E_{z_{\alpha}}(\mathcal{P}) \mathcal{H} \text { and } \operatorname{Ran}\left(W_{\alpha o}^{+} E_{z_{\alpha}}(\mathcal{P})\right) \subset E_{z_{\alpha}}\left(\mathcal{P}_{\alpha}\right) \mathcal{H} \text {. }
$$

The Lemma B.5.1 of [4] implies then the second part of the assertion i).

Clearly $I=\sum_{\alpha \in \mathcal{L}} E_{Z_{\alpha}}(\mathcal{P})$ and by using Lemma 6.9 and the absence of singularly continuous spectrum, one obtains the equalities:

$$
\mathcal{H}_{\mathrm{ac}}(H)=\bigoplus_{\alpha \neq o} E_{z_{\alpha}}(\mathcal{P}) \mathcal{H}=\bigoplus_{\alpha \neq o} \operatorname{Ran} \Omega_{\alpha}^{+} .
$$


The statement iii) is due to the relation between $\mathcal{P}^{\alpha}$ and $\mathcal{P}_{\alpha}$. Since $H_{\alpha}=$ $-\boldsymbol{\Delta}^{\alpha^{\perp}} \otimes I+I \otimes H^{\alpha}$, and since the asymptotic velocity for the operator $-\boldsymbol{\Delta}_{j}$ is $P_{j}$, one has $\left(\mathcal{P}_{\alpha}\right)_{j}=P_{j}$ if $\alpha_{j} \neq 0$ and $\left(\mathcal{P}_{\alpha}\right)_{j}=\left(\mathcal{P}^{\alpha}\right)_{j}$ if $\alpha_{j}=0$. Then $E_{z_{\alpha}}\left(\mathcal{P}_{\alpha}\right)$ is obtained from the fact that $Z_{\alpha}$ is a cartesian product and from the relation $E_{\{0\}}\left(\mathcal{P}^{\alpha}\right)=E_{\mathrm{p}}\left(H^{\alpha}\right)$.

\section{Appendix}

Proof of Lemma 6.1. If $z \in \mathbb{C} \backslash \sigma(H)$, let us write $R(z)$ for $(H-z)^{-1}$. In order to express $\eta(H)$ in terms of the resolvent of $H$ we shall make use of the formula (6.1.26) of [1] for $m \geq 2$ :

$$
\begin{aligned}
\eta(H)= & \frac{1}{\pi} \Im \int_{\mathbb{R}}\left[\sum_{k=0}^{m-1} \frac{i^{k}}{k !} \eta^{(k)}(\lambda)\right] R(\lambda+i) d \lambda \\
& +\frac{1}{\pi} \Im \int_{\mathbb{R}} \int_{0}^{1} \frac{i^{m}}{(m-1) !} \mu^{m-1} \eta^{(m)}(\lambda) R(\lambda+i \mu) d \lambda d \mu .
\end{aligned}
$$

We shall also use the first resolvent equation

$$
R(\lambda+i \mu)=[I+(\lambda+i(\mu-1)) R(\lambda+i \mu)] R(i),
$$

and the fact that if $p, k, m$ are positive integers, with $m \geq p+1$, then the integrals

$$
\begin{gathered}
\int_{\mathbb{R}}\left|\eta^{(k)}(\lambda)\right|[1+|\lambda|]^{p} d \lambda \quad \text { and } \\
\int_{\mathbb{R}} \int_{0}^{1}\left|\eta^{(m)}(\lambda)\right|\left[1+(|\lambda|+|\mu-1|) \frac{1}{|\mu|}\right]^{p} \mu^{m-1} d \lambda d \mu
\end{gathered}
$$

are finite.

a) Let us replace $\eta(H)$ by $(25)$ in the statement i) and observe that

$$
\left[R(z), f\left(\frac{Q}{2 t}\right)\right]=\frac{1}{t} R(z)\left\{i P \cdot \nabla f\left(\frac{Q}{2 t}\right)-\frac{1}{4 t} \Delta f\left(\frac{Q}{2 t}\right)\right\} R(z) .
$$

By taking $z=\lambda+i \mu$ and using (26) for the resolvents on the r.h.s. of (28), one obtains that the norm of $\left[R(z), f\left(\frac{Q}{2 t}\right)\right](H+i)$ is less than

$$
\frac{1}{t}\left(1+(|\lambda|+|\mu-1|) \frac{1}{|\mu|}\right)^{2}\left\|R(i)\left\{i P \cdot \nabla f-\frac{1}{4 t} \Delta f\right\}\right\|\|R(i)(H+i)\| .
$$

Since the two norms are bounded uniformly in $t$ for $t \geq 1$, the statement i) is now easily proved by taking into account (27) with $m=3$. 
b) For the proof of the first statement of iii), let $\xi$ be an asymptotic localization function and $\delta>0$ such that $\xi\left( \pm \frac{x_{j}}{\delta}\right) f(x)=f(x)$ for all $x \in \mathbb{R}^{n}$. We start by replacing $\eta(H)$ by (25) in the statement iii). By using that $\xi(2 y) \xi^{\prime}(y)=\xi^{\prime}(y)$ for all $y \in \mathbb{R}$, some commutator calculations show that

$$
\begin{gathered}
{\left[P_{j}, R(z)\right] \xi\left( \pm \frac{Q_{j}}{2 \delta t}\right)=i R(z) \xi\left( \pm \frac{Q_{j}}{2 \delta t}\right) \nabla_{j} V(Q) R(z)} \\
\mp \frac{1}{\delta t} \xi^{\prime}\left( \pm \frac{Q_{j}}{2 \delta t}\right) R(z) \xi\left( \pm \frac{Q_{j}}{\delta t}\right) \nabla_{j} V(Q) R(z) P_{j} R(z)+\left(\frac{1}{2 \delta t}\right)^{2} \mathcal{R}
\end{gathered}
$$

where $\mathcal{R}$ is equal to

$$
\begin{aligned}
& i R \nabla_{j} V(Q) R \xi^{\prime \prime}\left( \pm \frac{Q_{j}}{2 \delta t}\right) R \\
& \quad+R\left\{-4 i P_{j} \xi^{\prime \prime}\left( \pm \frac{Q_{j}}{2 \delta t}\right) \pm \frac{1}{\delta t} \xi^{\prime \prime \prime}\left( \pm \frac{Q_{j}}{2 \delta t}\right)\right\} R \xi\left( \pm \frac{Q_{j}}{\delta t}\right) \nabla_{j} V(Q) R P_{j} R \\
& \quad+R \nabla_{j} V(Q) R\left\{-4 i \xi^{\prime \prime}\left( \pm \frac{Q_{j}}{2 \delta t}\right) P_{j} \mp \frac{1}{\delta t} \xi^{\prime \prime \prime}\left( \pm \frac{Q_{j}}{2 \delta t}\right)\right\} R P_{j} R .
\end{aligned}
$$

(we have written $R$ for $R(z)$ ). Again, by taking $z=\lambda+i \mu$ and using (26) for the resolvents of the first two terms on the r.h.s. of (29), one obtains that their norm is less than

$$
\begin{aligned}
& \left(1+(|\lambda|+|\mu-1|) \frac{1}{|\mu|}\right)^{2}\left\|R(i) \xi\left( \pm \frac{Q_{j}}{2 \delta t}\right) \nabla_{j} V(Q) R(i)\right\| \\
& \quad+c\left(1+(|\lambda|+|\mu-1|) \frac{1}{|\mu|}\right)^{3}\left\|R(i) \xi\left( \pm \frac{Q_{j}}{\delta t}\right) \nabla_{j} V(Q) R(i)\right\|\left\|P_{j} R(i)\right\|,
\end{aligned}
$$

where $c=\frac{1}{\delta}\left\|\xi^{\prime}\right\|_{L^{\infty}}$ is independent of $t, \eta$ and $\mu$ (one has used that $\frac{1}{t} \leq 1$ for all $t \geq 1$ ). Similarly, one can check that the norm of $\mathcal{R}$ is less than a polynomial in $\left(1+(|\lambda|+|\mu-1|) \frac{1}{|\mu|}\right)$ of order 4 with coefficients independent of $t, \lambda$ and $\mu$. One finishes the proof by taking into account (27) with $m=5$ and by observing that the two norms in (30) and the factor $\left(\frac{1}{2 \delta t}\right)^{2}$ in $(29)$ belong to $L^{1}((1, \infty), d t)$.

c) For the second statement of iii) let us denote by $\alpha$ the only element of $\mathcal{L}$ such that $\alpha \lessdot o$ and $\alpha_{j}= \pm 1$. One observes that $\left[P_{j}, V(Q)\right]=\left[P_{j}, V(Q)-\right.$ $\left.V^{\alpha}(Q)\right]$ since $V^{\alpha}$ does not depend on $x_{j}$. Hence, one has

$\left[P_{j}, R(z)\right]=\left\{R(z)\left(V(Q)-V^{\alpha}(Q)\right)\right\} P_{j} R(z)-R(z) P_{j}\left\{\left(V(Q)-V^{\alpha}(Q)\right) R(z)\right\}$.

Then, the same method and the same arguments already used in a) and b) may be applied. Since $\xi\left( \pm \frac{Q_{j}}{2 \delta t}\right)\left(V(Q)-V^{\alpha}(Q)\right) R(i) \in o\left(t^{0}\right)$ by definition of the cartesian potential $V$, one obtains that $\left[P_{j}, \eta(H)\right] f\left(\frac{Q}{2 t}\right) \in o\left(t^{0}\right)$. 
d) For the statement v), let us observe that

$$
\begin{aligned}
& \left(P_{j}+i\right) Q_{j} R(z)\left(1+Q^{2}\right)^{-1 / 2} \\
& \quad=\left(P_{j}+i\right) R(z) Q_{j}\left(1+Q^{2}\right)^{-1 / 2}-2 i\left(P_{j}+i\right) R(z) P_{j} R(z)\left(1+Q^{2}\right)^{-1 / 2} .
\end{aligned}
$$

In order to make the calculation of the commutator $\left[Q_{j}, R(z)\right]$ properly, one has invoked the invariance of $\mathcal{H}^{2}$ under the group $\left\{e^{i y Q_{j}}\right\}_{y \in \mathbb{R}}$ and the statement (a) of Theorem 6.3.4 of [1]. Then the end of the proof follows the scheme of the previous points.

e) For the statement ii), let $\delta>0$ and $\xi$ be an asymptotic localization function such that $\xi\left( \pm \frac{x_{j}}{\delta}\right) f(x)=f(x)$ for all $x \in \mathbb{R}^{n}$. One has

$$
\begin{gathered}
\eta(H) f\left(\frac{Q}{2 t}\right) \nabla_{j} V(Q) \eta(H)=f\left(\frac{Q}{2 t}\right) \eta(H) \xi\left( \pm \frac{Q_{j}}{2 \delta t}\right) \nabla_{j} V(Q) \eta(H) \\
+\left[\eta(H), f\left(\frac{Q}{2 t}\right)\right](\boldsymbol{\Delta}+i)(\boldsymbol{\Delta}+i)^{-1} \xi\left( \pm \frac{Q_{j}}{2 \delta t}\right) \nabla_{j} V(Q) \eta(H)
\end{gathered}
$$

One observes that $f\left(\frac{Q}{2 t}\right)$ and $\left[\eta(H), f\left(\frac{Q}{2 t}\right)\right](\boldsymbol{\Delta}+i)$ are norm bounded independently of $t$ for $t \geq 1$ (use $i$ ) for the second term). By taking (5) into account, one gets the expected result.

f) The proof of iv) is similar to the one given in the paragraph b) of Lemma 4.1. If $\delta>0$ and $\xi$ is an asymptotic localization function, one observes that $\left\{1-\prod_{\left\{j \mid \alpha_{j} \neq 0\right\}} \xi\left(\alpha_{j} \frac{x_{j}}{2 \delta t}\right)\right\}_{t \geq 1}$ is an approximate unit for the ideal $\left\langle J_{\alpha} \cdot C_{0}\left(\mathbb{R}^{n *}\right)\right\rangle$. Since $\eta(H)-\eta\left(H_{\alpha}\right)$ belongs to this ideal, it follows that $\lim _{t \rightarrow \infty}\left\|\prod_{\left\{j \mid \alpha_{j} \neq 0\right\}} \xi\left(\alpha_{j} \frac{Q_{j}}{2 \delta t}\right)\left(\eta(H)-\eta\left(H_{\alpha}\right)\right)\right\|=0$.

Proof of Lemma 6.2. Since the following inequality is satisfied for all $t \geq 1$ and all $\varphi \in \mathcal{H}^{2}$ :

$$
\left\langle\varphi, B^{*}(t) B(t) \varphi\right\rangle \leq \pm\langle\varphi, \mathbf{D} \Phi(t) \varphi\rangle-\langle\varphi, F(t) \varphi\rangle-\langle\varphi, L(t) \varphi\rangle
$$

one obtains that

$$
\begin{aligned}
\int_{1}^{\infty}\left\|B(t) e^{-i H t} \varphi\right\|^{2} d t \leq & \left|\int_{1}^{\infty}\left\langle e^{-i H t} \varphi, \mathbf{D} \Phi(t) e^{-i H t} \varphi\right\rangle d t\right| \\
& +\int_{1}^{\infty}\left|\left\langle e^{-i H t} \varphi, F(t) e^{-i H t} \varphi\right\rangle\right| d t+\|\varphi\|^{2} \int_{1}^{\infty}\|L(t)\| d t
\end{aligned}
$$

But one has noticed that $\left\langle e^{-i H t} \varphi, \mathbf{D} \Phi(t) e^{-i H t} \varphi\right\rangle=\frac{d}{d t}\left\langle e^{-i H t} \varphi, \Phi(t) e^{-i H t} \varphi\right\rangle$, and hence $\left|\int_{1}^{\infty}\left\langle e^{-i H t} \varphi, \mathbf{D} \Phi(t) e^{-i H t} \varphi\right\rangle d t\right| \leq 2\|\Phi\|_{L^{\infty}}\|\varphi\|^{2}$. The conclusion is now straightforward. 
Proof of Lemma 6.3. Let $\psi \in \mathcal{D}_{2}, \varphi \in \mathcal{D}_{1}$ and $1 \leq s<t$. One has

$$
\begin{aligned}
\mid\langle\psi & \left.\left\{e^{i H_{2} t} \Phi(t) e^{-i H_{1} t}-e^{i H_{2} s} \Phi(s) e^{-i H_{1} s}\right\} \varphi\right\rangle \mid \\
\leq & \int_{s}^{t}\left|\left\langle\psi, e^{i H_{2} \tau}\left(i H_{2} \Phi(\tau)-i \Phi(\tau) H_{1}+\frac{d}{d \tau} \Phi(\tau)\right) e^{-i H_{1} \tau} \varphi\right\rangle\right| d \tau \\
\leq & \sum_{k=1}^{N}\left(\int_{s}^{t}\left\|E_{k}(\tau) e^{-i H_{2} \tau} \psi\right\|^{2} d \tau\right)^{\frac{1}{2}}\left(\int_{s}^{t}\left\|F_{k}(\tau) e^{-i H_{1} \tau} \varphi\right\|^{2} d \tau\right)^{\frac{1}{2}} \\
& +\left(\int_{s}^{t}\|L(\tau)\| d \tau\right)\|\psi\|\|\varphi\| .
\end{aligned}
$$

Thus, one gets that

$$
\begin{aligned}
& \left\|e^{i H_{2} t} \Phi(t) e^{-i H_{1} t} \varphi-e^{i H_{2} s} \Phi(s) e^{-i H_{1} s} \varphi\right\| \\
& \quad=\sup _{\psi \in \mathcal{D}_{2},\|\psi\|=1}\left|\left\langle\psi,\left\{e^{i H_{2} t} \Phi(t) e^{-i H_{1} t}-e^{i H_{2} s} \Phi(s) e^{-i H_{1} s}\right\} \varphi\right\rangle\right| \\
& \leq c \sum_{k=1}^{N}\left(\int_{s}^{t}\left\|F_{k}(\tau) e^{-i H_{1} \tau} \varphi\right\|^{2} d \tau\right)^{\frac{1}{2}}+\left(\int_{s}^{t}\|L(\tau)\| d \tau\right)\|\varphi\| .
\end{aligned}
$$

Since (31) can be made arbitrarily small by choosing $s$ large enough, one gets the existence of $\mathrm{s}-\lim _{t \rightarrow \infty} e^{i H_{2} t} \Phi(t) e^{-i H_{1} t} \varphi$ for all $\varphi \in \mathcal{D}_{1}$, which implies the result of this lemma.

\section{Acknowledgements}

The author thanks M. Măntoiu for suggesting to him this work and for his helpful advice. He also expresses his gratitude to W. O. Amrein for critical reading of the text and for his constant support. He finally thanks the referee for pointing out some misprints.

\section{References}

[1] Amrein, W. O., Boutet de Monvel, A. and Georgescu, V., C 0 -Groups, Commutator Methods and Spectral Theory of N-Body Hamiltonians, Birkhäuser Verlag, 1996.

[2] Amrein, W. O., Măntoiu, M. and Purice, R., Propagation Properties for Schrödinger Operators Affiliated to Certain C*-Algebras, Ann. Henri Poincaré, 3 (2002), 1215-1232.

[3] Davies, E. B. and Simon, B., Scattering Theory for Systems with Different Spatial Asymptotics on the Left and Right, Comm. Math. Phys., 63 (1978), 277-301.

[4] Dereziński, J. and Gérard, C., Scattering Theory of Classical and Quantum N-Particle Systems, Springer-Verlag Berlin Heidelberg, 1997.

[5] Dermenjian, Y. and Iftimie, V., Nouveaux canaux de diffusion pour les milieux stratifiés perturbés, Preprint LATP (2002), Université de Provence, Marseille. 
[6] Froese, R. and Herbst, I., A New Proof of the Mourre Estimate, Duke Math. J., 49, No 4 (1982), 1075-1085.

[7] Georgescu, V. and Iftimovici, A., On the Spectral Analysis of Anisotropic Quantum Systems, unpublished.

[8] - Crossed Products of $\mathrm{C}^{*}$-Algebras and Spectral Analysis of Quantum Hamiltonians, Comm. Math. Phys., 228 (2002), 519-560.

[9] Graf, G. M., Asymptotic Completeness for N-Body Short-Range Quantum Systems: A New Proof, Comm. Math. Phys., 132 (1990), 73-101.

[10] Herbst, I. and Skibsted, E., Quantum Scattering for Potentials Independent of $|x|$ : Asymptotic Completeness for High and Low Energies, to appear in Comm. Partial Differential Equations.

[11] Kato, T., Perturbation Theory for Linear Operators, Springer-Verlag, 1966.

[12] Măntoiu, M., C*-Algebras, Dynamical Systems at Infinity and the Essential Spectrum of Generalized Schrödinger Operators, J. Reine Angew. Math., 550 (2002), 211-229.

[13] _ C*-Algebras and Topological Dynamics in Spectral Analysis, Conference Proceedings: Operator Algebras and Mathematical Physics, Constanta (July 2001), Theta, Bucharest (2003), 299-314.

[14] Mourre, E., Absence of Singular Continuous Spectrum for Certain Self-Adjoint Operators, Comm. Math. Phys., 78 (1981), 391-408.

[15] — Opérateurs conjugués et propriétés de propagation, Comm. Math. Phys., 91 (1983), 279-300.

[16] Perry, P., Sigal, I. M. and Simon, B., Spectral Analysis of N-Body Schrödinger Operators, Ann. Math., 114 (1981), 519-567.

[17] Reed, M. and Simon, B., Methods of Modern Mathematical Physics IV: Analysis of Operators, Academic Press, 1978.

[18] Sigal, I. M. and Soffer, A., The N-Particle Scattering Problem: Asymptotic Completeness for Short-Range Systems, Ann. Math., 126 (1987), 35-108.

[19] Takai, H., On a Duality for Crossed Products of C*-Algebras, J. Funct. Anal., 19 (1975), 25-39. 